\title{
TOPOLOGICAL GRADIENT FOR A FOURTH ORDER OPERATOR USED IN IMAGE ANALYSIS
}

\author{
Gilles Aubert ${ }^{1}$ And Audric Drogoul ${ }^{1}$
}

\begin{abstract}
This paper is concerned with the computation of the topological gradient associated to a fourth order Kirchhoff type partial differential equation and to a second order cost function. This computation is motivated by fine structure detection in image analysis. The study of the topological sensitivity is performed both in the cases of a circular inclusion and a crack.
\end{abstract}

Mathematics Subject Classification. 35J30, 49Q10, 49Q12, 94A08, $94 \mathrm{~A} 13$.

Received December 9, 2013. Revised November 24, 2014

Published online June 30, 2015.

\section{INTRODUCTION}

The notion of topological gradient which has been rigorously formalized in $[13,17]$ for shape optimization problems has a wide range of applications: structural mechanics, optimal design, inverse analysis and more recently image processing [6-8]. Roughly speaking the topological gradient approach performs as follows: let $\Omega$ be an open bounded set of $\mathbb{R}^{2}$ and $j(\Omega)=J\left(\Omega, u_{\Omega}\right)$ be a cost function where $u_{\Omega}$ is the solution of a given PDE on $\Omega$. For small $\epsilon \geq 0$, let $\Omega_{\epsilon}=\Omega \backslash \overline{x_{0}+\epsilon \omega}$, where $x_{0} \in \Omega$ and $\omega$ is a given subset of $\mathbb{R}^{2}$. The topological analysis provides an asymptotic expansion of $j\left(\Omega_{\epsilon}\right)$ as $\epsilon \rightarrow 0$. In most cases it takes the form:

$$
j\left(\Omega_{\epsilon}\right)=j(\Omega)+\epsilon^{2} \mathcal{I}\left(x_{0}\right)+o\left(\epsilon^{2}\right)
$$

$\mathcal{I}\left(x_{0}\right)$ is called the topological gradient at $x_{0}$.

Thus, in optimal design for example, if we want to minimize $j\left(\Omega_{\epsilon}\right)$ it would be preferable to create holes at points $x_{0}$ where $\mathcal{I}\left(x_{0}\right)$ is "the most negative". In practice, we keep points $x_{0}$ where the topological gradient is less than a given negative threshold. In image processing the choice of the cost function is guided by the aimed application. For example for detection or segmentation problems, we have to choose a cost function which blows up in a neighbourhood of the structure we want to detect. Thus removing from the initial domain such a neighbourhood implies a large variation of the cost function and so a large topological gradient. In [8] the method was applied for edge detection by studying the topological sensitivity of $j(\Omega)=\int_{\Omega}\left|\nabla u_{\Omega}\right|^{2} \mathrm{~d} x$ where $u_{\Omega}$ is the solution of a Laplace equation. For filament (or point) detection, the problematic is different. Indeed there is no jump of the image intensity across this type of structure which is of zero Lebesgue measure. Typically the intensity takes the value 1 on the fine structure and 0 elsewhere. It is known (see for example Steger [18]) that

Keywords and phrases. Topological gradient, fourth order PDE, fine structures, 2D imaging.

1 Université Nice Sophia Antipolis, CNRS, LJAD, UMR 7351, 06100 Nice, France. gaubert@unice.fr; drogoula@unice.fr 

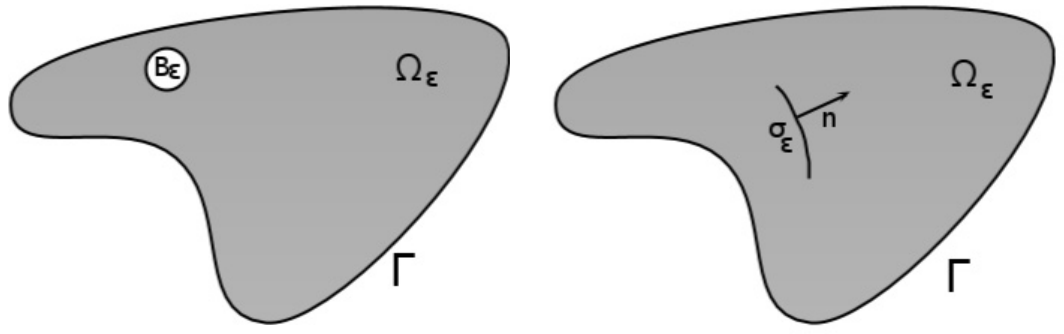

Figure 1. (a) Perforated domain, (b) cracked domain.

the gradient operator is not adapted since "it does not see" these structures. In this case we have to use a cost function defined from the Hessian matrix of a regularized version of the initial image. Inspired from the theory of thin plates ([9], Chap. 8), the main goal of this paper is to compute the topological gradient associated to the following model: if $\Omega$ denotes the image domain and if $f$ is the initial grey values image (the data, possibly degraded) we search for $u_{\Omega}$ as the solution of the fourth order PDE:

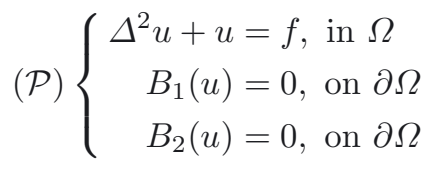

where $B_{1}(u)$ and $B_{2}(u)$ are natural boundary conditions to be specified in the next section (in fact we will study a more general model). The cost function is then defined as:

$$
J_{\Omega}(u)=\int_{\Omega}(\Delta u)^{2}+2(1-\nu)\left(\left(\frac{\partial^{2} u}{\partial x_{1} \partial x_{2}}\right)^{2}-\frac{\partial^{2} u}{\partial x_{1}^{2}} \frac{\partial^{2} u}{\partial x_{2}^{2}}\right),
$$

where $1<\nu<0$ is a parameter (the Poisson ratio). The link between $u_{\Omega}$ and $J_{\Omega}(u)$ is

$$
u_{\Omega}=\underset{u \in H^{2}(\Omega)}{\operatorname{argmin}} J_{\Omega}(u)+\|u-f\|_{L^{2}(\Omega)}^{2} .
$$

Let us notice that for $\nu=0$ the cost function simplifies as $J_{\Omega}(u)=\int_{\Omega}\left\|\nabla^{2} u\right\|_{2}^{2}$. This latter cost function has been used in the numerical companion paper [10] for detecting fine structures in 2D or 3D images (see also [11]).

In this work we compute the topological gradient associated to (1.2) and (1.3) when $\Omega_{\epsilon}=\Omega \backslash \overline{x_{0}+\epsilon B(0,1)}$ and $\Omega_{\epsilon}=\Omega \backslash \overline{x_{0}+\epsilon \sigma(n)}$, where $B(0,1)$ is the unit ball of $\mathbb{R}^{2}$ and $\sigma(n)$ is a straight segment with normal $n$ (a crack, see Fig. 1).

We warn the reader that the proofs are very technical and we only give the main steps. For the complete proofs and some results in 3D we refer the reader to [11]. The numerical analysis of the model as well numerous examples will be given in [10]. Most of the results of our work have been announced in [5].

Remark 1.1. The study of topological sensitivity for fourth order operators is not new. In [4], the authors in a different context, compute the topological gradient for the Kirchhoff plate bending in the case of a circular inclusion. Our model is simpler and we are able to give explicit expressions of the topological gradient both in the cases of circular inclusions and of cracks.

Remark 1.2. The link between the PDE and the cost function simplifies the topological gradient computation but it is easy to adapt the method for some other cost functions.

In the following, we denote by $\|u\|_{m, \Omega}=\|u\|_{H^{m}(\Omega)}$ (respectively $|u|_{m, \Omega}=|u|_{H^{m}(\Omega)}$ ) the norm (respectively the seminorm) on the Sobolev spaces $H^{m}(\Omega)$ and $\|u\|_{s, \Gamma}$ the norm on the fractional Sobolev space $H^{s}(\Gamma)$ with $\Gamma=\partial \Omega$. 
The outline of the paper is as follows. In Section 2 we define precisely the cost function and the variational problem. In Section 3 (respectively Sect. 4) we give the main steps of the computation of the topological gradient in the case of a circular inclusion (respectively of a crack). The paper ends with three appendices in which are developed details not given in Sections 3 and 4.

\section{Definition of The COST FUnCTION AND VARIATIONAL MODEL}

In this section we specify the general cost function and the variational model we want to study. Before, we give a lemma explaining why the natural operator we have to use in the cost function for detecting points and curves in 2D must be of second order.

\subsection{Detecting fine structures: what is the good operator?}

We denote by $\mathcal{D}\left(\mathbb{R}^{2}\right)$ the space of $C^{\infty}$ - functions with compact support in $\mathbb{R}^{2}$ and $\mathcal{D}^{\prime}\left(\mathbb{R}^{2}\right)$ the space of distributions on $\mathbb{R}^{2}$. We refer the reader to [11] for the proof of the following lemma.

Lemma 2.1. Let $\varphi: \mathbb{R}^{2} \longrightarrow \mathbb{R}$ be a Lipschitz continuous function, and let $\left(g_{h}\right)_{h>0}$ be a sequence of functions defined by

$$
g_{h}(x)=\frac{1}{\theta_{1}(h)} \mathrm{e}^{-\frac{\varphi^{2}(x)}{\theta_{2}(h)}} \quad \text { where } \theta_{i}: \mathbb{R}^{+} \longrightarrow \mathbb{R}^{+} \text {and } \lim _{h \rightarrow 0} \theta_{i}(h)=0 .
$$

(i) Let $a \in \mathbb{R}^{2}$, by setting $\varphi(x)=\|x-a\|, \theta_{1}(h)=\pi h$ and $\theta_{2}(h)=h$ then

$$
g_{h} \underset{h \rightarrow 0}{\longrightarrow} \delta_{a} \text {, in } \mathcal{D}^{\prime}\left(\mathbb{R}^{2}\right) \text {. }
$$

Besides we have

$$
\nabla g_{h}(a)=[0,0]^{T}, \quad \nabla^{2} g_{h}(a)=-\frac{2}{\pi h^{2}} I,
$$

where I denotes the identity in $\mathbb{R}^{2}$.

(ii) Let $\Gamma$ be a smooth closed curve or a smooth infinite curve of $\mathbb{R}^{2}$ delimiting two sub-domains $\mathbb{R}_{\Gamma}^{2-}$ and $\mathbb{R}_{\Gamma}^{2+}$ forming a partition of $\mathbb{R}^{2}$. Let $\varphi$ be the signed distance to $\Gamma$ defined by:

$$
\varphi(x)=\operatorname{dist}\left(x, \mathbb{R}_{\Gamma}^{2-}\right)-\operatorname{dist}\left(x, \mathbb{R}_{\Gamma}^{2+}\right) .
$$

We denote by $\mathbb{R}_{\Gamma}^{2+}$ (resp. $\mathbb{R}_{\Gamma}^{2-}$ ), the sub-domain $\{\varphi>0\}$ (resp. $\left.\{\varphi<0\}\right)$. Taking the following scalings: $\theta_{1}(h)=\sqrt{\pi h}$ and $\theta_{2}(h)=h$, we have

$$
g_{h} \underset{h \rightarrow 0}{\longrightarrow} \delta_{\Gamma}, \text { in } \mathcal{D}^{\prime}\left(\mathbb{R}^{2}\right)
$$

Besides for all $x \in \Gamma$ we have

$$
\nabla g_{h}(x)=[0,0]^{T}, \quad \operatorname{spec}\left(\nabla^{2} g_{h}(x)\right)=\left\{-\frac{2}{h^{3 / 2}}, 0\right\},
$$

where spec $(M)$ denotes the eigenvalues of the matrix $M$. The associated eigenvectors to $\nabla^{2} g_{h}$ on $\Gamma$ are $\left(\nabla \varphi(x), \nabla \varphi(x)^{\perp}\right)$, where $\nabla \varphi(x)=n(x)$.

So this lemma shows heuristically that the gradient "does not see" fines structures in $\mathbb{R}^{2}$ (points and filaments). On the other hand second derivatives are singular on these structures. 


\subsection{Definition of the cost function and the fourth order PDE}

We give now the general cost function we are going to study. The cost function is more general than (1.3). It is defined by:

$$
J_{\Omega}(u)=\int_{\Omega}(\Delta u)^{2}+2(1-\nu)\left(\left(\frac{\partial^{2} u}{\partial x_{1} \partial x_{2}}\right)^{2}-\frac{\partial^{2} u}{\partial x_{1}^{2}} \frac{\partial^{2} u}{\partial x_{2}^{2}}\right)+\gamma|\nabla u|^{2} \mathrm{~d} x, 0<\nu<1, \gamma \geq 0
$$

\section{Remark 2.2.}

(i) When $\gamma=0$ we retrieve (1.3).

(ii) We check easily that $J_{\Omega}$ :

$$
J_{\Omega}(u) \geq(1-\nu)|u|_{H^{2}(\Omega)}^{2}+\gamma|u|_{H^{1}(\Omega)}^{2}, \forall u \in H^{2}(\Omega) .
$$

For small $\epsilon>0$, let (a) $\Omega_{\epsilon}=\Omega \backslash \overline{\left\{x_{0}+\epsilon \omega\right\}}$ or (b) $\Omega_{\epsilon}=\Omega \backslash \overline{\left\{x_{0}+\epsilon \sigma(n)\right\}}$, where $x_{0} \in \Omega, \omega=B(O, 1)$ is the unit ball of $\mathbb{R}^{2}$ and $\sigma(n)$ is a straight segment centered at the origin and with normal $n$ (a crack). We introduce the bilinear and linear forms:

$$
\begin{aligned}
a_{\epsilon}(u, v) & =\int_{\Omega_{\epsilon}} \Delta u \Delta v+(1-\nu)\left(2 \frac{\partial^{2} u}{\partial x_{1} \partial x_{2}} \frac{\partial^{2} v}{\partial x_{1} \partial x_{2}}-\frac{\partial^{2} u}{\partial x_{1}^{2}} \frac{\partial^{2} v}{\partial x_{2}^{2}}-\frac{\partial^{2} u}{\partial x_{2}^{2}} \frac{\partial^{2} v}{\partial x_{1}^{2}}\right)+\gamma \nabla u . \nabla v+u v, \\
l_{\epsilon}(v) & =\int_{\Omega_{\epsilon}} f v .
\end{aligned}
$$

Thanks to Lax-Milgram lemma, it is easy to prove that for $\epsilon \geq 0$ fixed there exists a unique $u_{\epsilon} \in H^{2}\left(\Omega_{\epsilon}\right)$ such that

$$
a_{\epsilon}\left(u_{\epsilon}, v\right)=l_{\epsilon}(v), \forall v \in H^{2}\left(\Omega_{\epsilon}\right) .
$$

This solution $u_{\epsilon}$ necessarily satisfies the Euler equation:

$$
\left(\mathcal{P}_{\epsilon}\right)\left\{\begin{aligned}
\Delta^{2} u_{\epsilon}-\gamma \Delta u_{\epsilon}+u_{\epsilon} & =f, \text { on } \Omega_{\epsilon} \\
B_{1}\left(u_{\epsilon}\right)-\gamma \partial_{n} u_{\epsilon} & =0, \text { on } \partial \Omega_{\epsilon} \\
B_{2}\left(u_{\epsilon}\right) & =0, \text { on } \partial \Omega_{\epsilon}
\end{aligned}\right.
$$

where

and

$$
B_{1}(u)=\partial_{n}(\Delta u)-(1-\nu) \partial_{\sigma}\left(n_{1} n_{2}\left(\frac{\partial^{2} u}{\partial x_{1}^{2}}-\frac{\partial^{2} u}{\partial x_{2}^{2}}\right)-\left(n_{1}^{2}-n_{2}^{2}\right) \frac{\partial^{2} u}{\partial x_{1} \partial x_{2}}\right)
$$

$$
B_{2}(u)=\nu \Delta u+(1-\nu)\left(n_{1}^{2} \frac{\partial^{2} u}{\partial x_{1}^{2}}+n_{2}^{2} \frac{\partial^{2} u}{\partial x_{2}^{2}}+2 n_{1} n_{2} \frac{\partial^{2} u}{\partial x_{1} \partial x_{2}}\right),
$$

where $f \in L^{2}\left(\Omega_{\epsilon}\right) ; \boldsymbol{n}=\left(n_{1}, n_{2}\right)$ is the outward normal to the domain, and $\boldsymbol{\sigma}=\left(\sigma_{1}, \sigma_{2}\right)$ is the tangent vector such that $(\boldsymbol{n}, \boldsymbol{\sigma})$ forms an orthonormal basis.

\section{Remark 2.3.}

(i) When $\gamma=0,(2.4)$ is well-posed by using Gagliardo-Nirenberg inequalities [1,15]. The computation of the topological gradient is the same as in the case $\gamma \neq 0$.

(ii) We have the following relation: $a_{\epsilon}(u, u)=J_{\Omega_{\epsilon}}(u)+\|u\|_{0, \Omega_{\epsilon}}^{2}$.

We denote by $\mathbb{P}_{1}$ the set of polynomial of degree less or equal than 1 , and by $C$ all constants not depending on $\epsilon$. Finally, we will use the quotient space $H^{m}(\Omega) / \mathbb{P}_{1}$ which is the set of $H^{m}(\Omega)$ functions defined up to a polynomial of degree less or equal than 1 . In the paper, we study independently the case of a domain perforated by a ball and the case of a cracked domain. 


\section{Computation of the topological gradient in the CASE of the BalL}

\subsection{Notations and statement of the problem}

Let $x_{0} \in \Omega$ and $B(O, 1)$ the unit ball. We define $\Omega_{\epsilon}=\Omega \backslash \overline{x_{0}+\epsilon B(O, 1)}$ with $x_{0}$ and $\epsilon$ chosen such that the perturbation does not touch the border. By denoting $B=B\left(x_{0}, 1\right)$ and $B_{\epsilon}=B\left(x_{0}, \epsilon\right)$ we have $\partial \Omega_{\epsilon}=\partial B_{\epsilon} \cup \Gamma$ $(\Gamma=\partial \Omega)$.

Problem $\left(\mathcal{P}_{\epsilon}\right)$ rewrites as

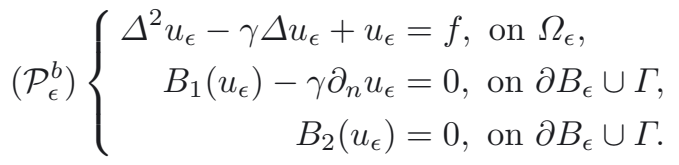

Let $v \in H^{2}\left(\Omega_{\epsilon}\right)$, and $u_{\epsilon}$ the solution of $\left(\mathcal{P}_{\epsilon}^{b}\right)$. By a classical regularity result, $u_{\epsilon} \in H^{4}\left(\Omega_{\epsilon}\right)$. Then by using integration by parts on $\Omega_{\epsilon}$, and ([9], p. 376), we get from (2.4) and (2.5):

$$
\begin{aligned}
\int_{\Omega_{\epsilon}} f v & =\int_{\Omega_{\epsilon}}\left(\Delta^{2} u_{\epsilon}-\gamma \Delta u_{\epsilon}+u_{\epsilon}\right) v \\
& =a_{\epsilon}\left(u_{\epsilon}, v\right)-\int_{B_{\epsilon}}\left(\left(B_{1}\left(u_{\epsilon}\right)-\gamma \partial_{n} u_{\epsilon}\right) v-B_{2}\left(u_{\epsilon}\right) \partial_{n} v\right)+\int_{\Gamma}\left(\left(B_{1}\left(u_{\epsilon}\right)-\gamma \partial_{n} u_{\epsilon}\right) v-B_{2}\left(u_{\epsilon}\right) \partial_{n} v\right) .
\end{aligned}
$$

To simplify notations we suppose that $x_{0} \equiv 0$.

Now to compute the topological gradient, we have to estimate the leading term when $\epsilon \rightarrow 0$ in the difference $J_{\epsilon}\left(u_{\epsilon}\right)-J_{0}\left(u_{0}\right)$. Using equations satisfied by $u_{\epsilon}$ and $u_{0}$ we have

$$
J_{\epsilon}\left(u_{\epsilon}\right)-J_{0}\left(u_{0}\right)=\int_{\Omega_{\epsilon}}\left(f-2 u_{0}\right)\left(u_{\epsilon}-u_{0}\right)-\int_{\Omega_{\epsilon}}\left(u_{\epsilon}-u_{0}\right)^{2}-\int_{B_{\epsilon}}\left(f-u_{0}\right) u_{0} .
$$

Let us denote $L_{\epsilon}: H^{2}\left(\Omega_{\epsilon}\right) \longrightarrow \mathbb{R}$ the linear map

$$
L_{\epsilon}(u)=\int_{\Omega_{\epsilon}}\left(f-2 u_{0}\right) u, \forall u \in H^{2}\left(\Omega_{\epsilon}\right),
$$

and

$$
\mathcal{J}_{\epsilon}=-\int_{\Omega_{\epsilon}}\left(u_{\epsilon}-u_{0}\right)^{2}-\int_{B_{\epsilon}}\left(f-u_{0}\right) u_{0} .
$$

The first step for evaluating (3.2) is to introduce $v_{\epsilon}$ the unique solution of the adjoint problem (see [2] and [17])

$$
a_{\epsilon}\left(u, v_{\epsilon}\right)=-L_{\epsilon}(u), \forall u \in H^{2}\left(\Omega_{\epsilon}\right) .
$$

From (3.5) and (3.4) we rewrite (3.2)

$$
\begin{aligned}
J_{\epsilon}\left(u_{\epsilon}\right)-J_{0}\left(u_{0}\right) & =-a_{\epsilon}\left(u_{\epsilon}-u_{0}, v_{\epsilon}\right)+\mathcal{J}_{\epsilon}=-l_{\epsilon}\left(v_{\epsilon}\right)+a_{\epsilon}\left(u_{0}, v_{\epsilon}\right)+\mathcal{J}_{\epsilon} \\
& =-\int_{\Omega_{\epsilon}} f v_{\epsilon}+\int_{\Omega_{\epsilon}}\left(\Delta^{2} u_{0}-\gamma \Delta u_{0}+u_{0}\right) v_{\epsilon}+\int_{\partial B_{\epsilon}}\left(B_{1}\left(u_{0}\right)-\gamma \partial_{n} u_{0}\right) v_{\epsilon}-B_{2}\left(u_{0}\right) \partial_{n} v_{\epsilon}+\mathcal{J}_{\epsilon} \\
& =\int_{\partial B_{\epsilon}}\left(B_{1}\left(u_{0}\right)-\gamma \partial_{n} u_{0}\right) v_{\epsilon}-B_{2}\left(u_{0}\right) \partial_{n} v_{\epsilon}+\mathcal{J}_{\epsilon} .
\end{aligned}
$$

Then we set $w_{\epsilon}=v_{\epsilon}-v_{0}$, where $v_{0}$ is the solution (3.5) with $\epsilon=0,\left(\Omega_{0}=\Omega\right)$, thus we rewrite

$$
J_{\epsilon}\left(u_{\epsilon}\right)-J_{0}\left(u_{0}\right)=\int_{\partial B_{\epsilon}}\left(B_{1}\left(u_{0}\right)-\gamma \partial_{n} u_{0}\right) v_{0}-B_{2}\left(u_{0}\right) \partial_{n} v_{0}+\int_{\partial B_{\epsilon}}\left(B_{1}\left(u_{0}\right)-\gamma \partial_{n} u_{0}\right) w_{\epsilon}-B_{2}\left(u_{0}\right) \partial_{n} w_{\epsilon}+\mathcal{J}_{\epsilon} .
$$

Now we express the difference $J_{\epsilon}\left(u_{\epsilon}\right)-J_{0}\left(u_{0}\right)$ as a sum of more simple terms. 
For $\varphi_{1} \in H^{3 / 2}\left(\partial B_{\epsilon}\right), \varphi_{2} \in H^{1 / 2}\left(\partial B_{\epsilon}\right)$ let $l_{\epsilon}^{\varphi_{1}, \varphi_{2}} \in H^{2}\left(B_{\epsilon}\right)$ the solution of the problem

$$
\left\{\begin{aligned}
\Delta^{2} l_{\epsilon}^{\varphi_{1}, \varphi_{2}} & =0, \text { on } B_{\epsilon} \\
l_{\epsilon}^{\varphi_{1}, \varphi_{2}} & =\varphi_{1}, \text { on } \partial B_{\epsilon} \\
\partial_{n} l_{\epsilon}^{\varphi_{1}, \varphi_{2}} & =\varphi_{2}, \text { on } \partial B_{\epsilon} .
\end{aligned}\right.
$$

For $u \in H^{2}\left(\Omega_{\epsilon}\right)$ we denote by $l_{\epsilon}^{u}$ the function $l_{\epsilon}^{u, \partial_{n} u}$, and for $\epsilon=1$ by $l^{\varphi_{1}, \varphi_{2}}$ the function $l_{1}^{\varphi_{1}, \varphi_{2}}$. The difference becomes:

$$
\begin{aligned}
J_{\epsilon}\left(u_{\epsilon}\right)-J_{0}\left(u_{0}\right) & =\int_{\partial B_{\epsilon}}\left(B_{1}\left(u_{0}\right)-\gamma \partial_{n} u_{0}\right) v_{0}-B_{2}\left(u_{0}\right) \partial_{n} v_{0}+\int_{\partial B_{\epsilon}}\left(B_{1}\left(u_{0}\right)-\gamma \partial_{n} u_{0}\right) l_{\epsilon}^{w_{\epsilon}}-B_{2}\left(u_{0}\right) \partial_{n} l_{\epsilon}^{w_{\epsilon}}+\mathcal{J}_{\epsilon} \\
& =\mathcal{L}_{\epsilon}+\mathcal{K}_{\epsilon}+\mathcal{J}_{\epsilon}
\end{aligned}
$$

where

$$
\mathcal{K}_{\epsilon}=\int_{\partial B_{\epsilon}}\left(B_{1}\left(u_{0}\right)-\gamma \partial_{n} u_{0}\right) l_{\epsilon}^{w_{\epsilon}}-B_{2}\left(u_{0}\right) \partial_{n} l_{\epsilon}^{w_{\epsilon}}, \quad \mathcal{L}_{\epsilon}=\int_{\partial B_{\epsilon}}\left(B_{1}\left(u_{0}\right)-\gamma \partial_{n} u_{0}\right) v_{0}-B_{2}\left(u_{0}\right) \partial_{n} v_{0} .
$$

Let $\widetilde{u_{0}}(x)=u_{0}(x)-u_{0}(0)-\nabla u_{0}(0) \cdot x$, it is straightforward that

$$
\mathcal{K}_{\epsilon}=\int_{\partial B_{\epsilon}}\left(B_{1}\left(\widetilde{u_{0}}\right)-\gamma \partial_{n} u_{0}\right) l_{\epsilon}^{w_{\epsilon}}-B_{2}\left(\widetilde{u_{0}}\right) \partial_{n} l_{\epsilon}^{w_{\epsilon}} .
$$

Then integration by parts (see [9], p. 376) gives

$$
\mathcal{K}_{\epsilon}=\int_{B_{\epsilon}} \Delta^{2} \widetilde{u_{0}} l_{\epsilon}^{w_{\epsilon}}-b_{\epsilon}\left(\widetilde{u_{0}}, l_{\epsilon}^{w_{\epsilon}}\right)-\int_{B_{\epsilon}} \gamma\left(\Delta u_{0} l_{\epsilon}^{w_{\epsilon}}-\nabla u_{0} . \nabla l_{\epsilon}^{w_{\epsilon}}\right)
$$

where $b_{\epsilon}(u, v)$ is the bilinear form associated to (3.6) and defined by

$$
b_{\epsilon}(u, v)=\int_{B_{\epsilon}} \Delta u \Delta v+(1-\nu)\left(2 \frac{\partial^{2} u}{\partial x_{1} \partial x_{2}} \frac{\partial^{2} v}{\partial x_{1} \partial x_{2}}-\frac{\partial^{2} u}{\partial x_{1}^{2}} \frac{\partial^{2} v}{\partial x_{2}^{2}}-\frac{\partial^{2} u}{\partial x_{2}^{2}} \frac{\partial^{2} v}{\partial x_{1}^{2}}\right) .
$$

Integration by parts again gives:

$$
\mathcal{K}_{\epsilon}=\int_{B_{\epsilon}}\left(\Delta^{2} u_{0}-\gamma \Delta u_{0}\right) l_{\epsilon}^{w_{\epsilon}}+\gamma \nabla u_{0} . \nabla l_{\epsilon}^{w_{\epsilon}}-\int_{B_{\epsilon}} \underbrace{\Delta^{2} l_{\epsilon}^{w_{\epsilon}}}_{=0} \widetilde{u_{0}}+\int_{\partial B_{\epsilon}} B_{1}\left(l_{\epsilon}^{w_{\epsilon}}\right) \widetilde{u_{0}}-B_{2}\left(l_{\epsilon}^{w_{\epsilon}}\right) \partial_{n} \widetilde{u_{0}} .
$$

In a similar manner

$$
\mathcal{L}_{\epsilon}=\int_{B_{\epsilon}}\left(\Delta^{2} u_{0}-\gamma \Delta u_{0}\right) v_{0}+\gamma \nabla u_{0} \cdot \nabla v_{0}-\int_{B_{\epsilon}} \Delta^{2} v_{0} \widetilde{u_{0}}+\int_{\partial B_{\epsilon}} B_{1}\left(v_{0}\right) \widetilde{u_{0}}-B_{2}\left(v_{0}\right) \partial_{n} \widetilde{u_{0}} .
$$

We set $F=\left(f-2 u_{0}\right)$, thus we have in the distributional sense for $\epsilon \rightarrow 0$ :

$$
\Delta^{2} v_{0}-\gamma \Delta v_{0}+v_{0}=-F \text { in } \mathcal{D}^{\prime}\left(B_{\epsilon}\right) .
$$

From (3.7)-(3.10) we get

$$
\begin{aligned}
\mathcal{L}_{\epsilon}+\mathcal{K}_{\epsilon}= & \int_{B_{\epsilon}}\left(f-u_{0}\right) v_{0}+\gamma \nabla u_{0} \cdot \nabla v_{0}+\int_{B_{\epsilon}}\left(F-\gamma \Delta v_{0}+v_{0}\right) \widetilde{u_{0}}+\int_{B_{\epsilon}}\left(\Delta^{2} u_{0}-\gamma \Delta u_{0}\right) l_{\epsilon}^{w_{\epsilon}} \\
& +\gamma \nabla u_{0} \cdot \nabla l_{\epsilon}^{w_{\epsilon}}+\int_{\partial B_{\epsilon}}\left(B_{1}\left(l_{\epsilon}^{w_{\epsilon}}\right)+B_{1}\left(v_{0}\right)\right) \widetilde{u_{0}}-\int_{\partial B_{\epsilon}}\left(B_{2}\left(l_{\epsilon}^{w_{\epsilon}}\right)+B_{2}\left(v_{0}\right)\right) \partial_{n} \widetilde{u_{0}} \\
= & \int_{B_{\epsilon}}\left(f-u_{0}\right) v_{0}+\gamma \nabla u_{0} \cdot \nabla v_{0}+J_{A}-J_{B}+\mathcal{E}_{1}+\mathcal{E}_{2}+\mathcal{E}_{3},
\end{aligned}
$$


where

$$
\begin{aligned}
& J_{A}=\int_{\partial B_{\epsilon}}\left(B_{1}\left(l_{\epsilon}^{w_{\epsilon}}\right)+B_{1}\left(v_{0}\right)\right) \widetilde{u_{0}}, \quad J_{B}=\int_{\partial B_{\epsilon}}\left(B_{2}\left(l_{\epsilon}^{w_{\epsilon}}\right)+B_{2}\left(v_{0}\right)\right) \partial_{n} \widetilde{u_{0}} \\
& \mathcal{E}_{1}=\int_{B_{\epsilon}}\left(F-\gamma \Delta v_{0}+v_{0}\right) \widetilde{u_{0}}, \quad \mathcal{E}_{2}=\int_{B_{\epsilon}}\left(\Delta^{2} u_{0}-\gamma \Delta u_{0}\right) l_{\epsilon}^{w_{\epsilon}}, \quad \mathcal{E}_{3}=\int_{B_{\epsilon}} \gamma \nabla u_{0} . \nabla l_{\epsilon}^{w_{\epsilon}} .
\end{aligned}
$$

In the next section, we will show that $\mathcal{E}_{1}, \mathcal{E}_{2}$ and $\mathcal{E}_{3}$ are $\backsim o\left(\epsilon^{2}\right)$ and that $J_{A}$ and $J_{B}$ are $\backsim O\left(\epsilon^{2}\right)$. Before we establish the asymptotic expansion of $B_{1}\left(v_{0}\right), B_{2}\left(v_{0}\right)$ and $w_{\epsilon}$.

\subsection{Estimates of $B_{1}\left(v_{0}\right)(x)$ and $B_{2}\left(v_{0}\right)(x)$ for $x \in \partial B_{\epsilon}$}

Proposition 3.1. Suppose $v_{0}$ regular, then when $\epsilon \rightarrow 0$ we have the following boundary expansions:

$$
B_{1}\left(v_{0}\right)(\epsilon X)=-\frac{g_{1}(X)}{\epsilon}+O(1), \quad B_{2}\left(v_{0}\right)(\epsilon X)=-g_{2}(X)+O(\epsilon),
$$

where setting $X=(\cos (\theta), \sin (\theta)) \in \partial B$

$$
\begin{aligned}
& g_{1}(X)=g_{1}(\theta)=(1-\nu)\left(\frac{\partial^{2} v_{0}}{\partial x_{1}^{2}}(0)-\frac{\partial^{2} v_{0}}{\partial x_{2}^{2}}(0)\right) \cos (2 \theta)+2(1-\nu) \frac{\partial^{2} v_{0}}{\partial x_{1} \partial x_{2}}(0) \sin (2 \theta), \\
& g_{2}(X)=g_{2}(\theta)=-\frac{(1+\nu)}{2} \Delta v_{0}(0)-\frac{(1-\nu)}{2}\left(\frac{\partial^{2} v_{0}}{\partial x_{1}^{2}}(0)-\frac{\partial^{2} v_{0}}{\partial x_{2}^{2}}(0)\right) \cos (2 \theta)-(1-\nu) \frac{\partial^{2} v_{0}}{\partial x_{1} \partial x_{2}}(0) \sin (2 \theta) .
\end{aligned}
$$

Proof. It suffices to expand $B_{1}\left(v_{0}\right)(\epsilon X)$ and $B_{2}\left(v_{0}\right)(\epsilon X)$ around $\epsilon=0$ by using Taylor formula (see [11] for details).

\subsection{Asymptotic expansion of $w_{\epsilon}$}

We recall that $w_{\epsilon}=v_{\epsilon}-v_{0}$ is the solution of:

$$
\left(\mathcal{Q}_{\epsilon}^{b}\right)\left\{\begin{aligned}
\Delta^{2} w_{\epsilon}-\gamma \Delta w_{\epsilon}+w_{\epsilon} & =0, \text { on } \Omega_{\epsilon}, \\
B_{1}\left(w_{\epsilon}\right)-\gamma \partial_{n} w_{\epsilon} & =-B_{1}\left(v_{0}\right)+\gamma \partial_{n} v_{0}, \text { on } \partial B_{\epsilon}, \\
B_{2}\left(w_{\epsilon}\right) & =-B_{2}\left(v_{0}\right), \text { on } \partial B_{\epsilon}, \\
B_{1}\left(w_{\epsilon}\right)-\gamma \partial_{n} w_{\epsilon} & =0, \text { on } \Gamma \\
B_{2}\left(w_{\epsilon}\right) & =0, \text { on } \Gamma .
\end{aligned}\right.
$$

We denote by $B^{\prime}$ the exterior domain $\mathbb{R}^{2} \backslash \bar{B}$, and we introduce the weighted Sobolev space [14]:

$$
W^{2}\left(B^{\prime}\right)=\left\{u, \frac{u}{\left(1+r^{2}\right) \log \left(2+r^{2}\right)} \in L^{2}\left(B^{\prime}\right), \frac{\nabla u}{\left(1+r^{2}\right)^{1 / 2} \log \left(2+r^{2}\right)} \in L^{2}\left(B^{\prime}\right), \nabla^{2} u \in L^{2}\left(B^{\prime}\right)\right\},
$$

where $r=|x|$. We denote by $W^{2}\left(B^{\prime}\right) / \mathbb{P}_{1}$ the set of functions $W^{2}\left(B^{\prime}\right)$ defined up to a polynomial of degree less or equal than 1 . To estimate $w_{\epsilon}$, we introduce (see $\left.[3,17]\right)$ the following exterior problem

$$
\left(\mathcal{P}_{\text {ext }}\right)\left\{\begin{array}{c}
\Delta^{2} P=0, \text { on } B^{\prime}, \\
B_{1}(P)=g_{1}, \text { on } \partial B, \\
B_{2}(P)=g_{2}, \text { on } \partial B,
\end{array}\right.
$$


where $g_{1} \in H^{-3 / 2}(\partial B)$ and $g_{2} \in H^{-1 / 2}(\partial B)$ are given in Proposition 3.1. From Theorem B.3 (Appendix B), we deduce that problem $\left(\mathcal{P}_{\text {ext }}\right)$ admits a unique solution $P \in W^{2}\left(B^{\prime}\right) / \mathbb{P}_{1}$ and $P$ can be written as the sum of simple and double layers potential:

$$
P(x)=\int_{\partial B} \lambda_{1}(y) E(x-y) \mathrm{d} \sigma(y)+\int_{\partial B} \lambda_{2}(y) \partial_{n_{y}}(E(x-y)) \mathrm{d} \sigma(y)
$$

where $\lambda_{1}$ and $\lambda_{2}$ are densities that we can determine in function of the boundary data; $E(x)$ denotes the bilaplacian fundamental solution:

$$
E(x)=-\frac{1}{8 \pi}|x|^{2} \log (|x|)
$$

From Theorem B.3 (Appendix B) and Proposition 3.1 we get

$$
\lambda_{1}=\alpha \cos (2 \theta)+\beta \sin (2 \theta), \quad \lambda_{2}=c+a \cos (2 \theta)+b \sin (2 \theta)
$$

where

$$
\begin{aligned}
& a=-2 \frac{1-\nu}{3+\nu}\left(\frac{\partial^{2} v_{0}}{\partial x_{1}^{2}}(0)-\frac{\partial^{2} v_{0}}{\partial x_{1}^{2}}(0)\right), \quad b=-4 \frac{1-\nu}{3+\nu} \frac{\partial^{2} v_{0}}{\partial x_{1} \partial x_{2}}(0), \quad c=-\frac{1+\nu}{1-\nu} \Delta v_{0}(0), \\
& \alpha=2 a, \quad \beta=2 b .
\end{aligned}
$$

Then from Lemma B.6 (Appendix B), we prove that $w_{\epsilon}=\epsilon^{2} P\left(\frac{x}{\epsilon}\right)+e_{\epsilon}$ with $\left\|e_{\epsilon}\right\|_{2, \Omega_{\epsilon}}=O\left(-\epsilon^{2} \log (\epsilon)\right)$. In the next subsection we estimate $J_{A}, J_{B}, \mathcal{E}_{1}, \mathcal{E}_{2}$ and $\mathcal{E}_{3}$.

\subsection{Estimates of $J_{A}, J_{B}, \mathcal{E}_{1}, \mathcal{E}_{2}$ and $\mathcal{E}_{3}$}

Lemma 3.2. Let $J_{A}, J_{B}, \mathcal{E}_{1}, \mathcal{E}_{2}$ and $\mathcal{E}_{3}$ given in (3.12), we have the following estimates

$$
\begin{aligned}
& J_{A}=\epsilon^{2} \frac{1}{2} \int_{\partial B} \lambda_{1}(y) \nabla^{2} u_{0}(0) y \cdot y \mathrm{~d} \sigma(y)+o\left(\epsilon^{2}\right), \quad J_{B}=-\epsilon^{2} \int_{\partial B} \lambda_{2}(y) \nabla^{2} u_{0}(0) y \cdot y \mathrm{~d} \sigma(y)+o\left(\epsilon^{2}\right), \\
& \mathcal{E}_{1}=O\left(\epsilon^{3}\right), \quad \mathcal{E}_{2}=O\left(-\epsilon^{3} \log (\epsilon)\right), \quad \mathcal{E}_{3}=O\left(-\epsilon^{3} \log (\epsilon)\right) .
\end{aligned}
$$

Proof. From the linearity of the solution of (3.6), by using the jump relations (B.7) given in Theorem B.3 (Appendix B) and the asymptotic expansion of $w_{\epsilon}$ we get

$$
\begin{aligned}
J_{A} & =\int_{\partial B_{\epsilon}}\left(B_{1}\left(v_{0}\right)+B_{1}\left(l_{\epsilon}^{\epsilon^{2} P\left(\frac{x}{\epsilon}\right)+e_{\epsilon}}\right)\right) \widetilde{u_{0}}=\epsilon \int_{\partial B}\left(B_{1}\left(v_{0}\right)(\epsilon X)+\frac{1}{\epsilon} B_{1}\left(l^{P}\right)(X)\right) \widetilde{u_{0}}(\epsilon X) \mathrm{d} \sigma(X)+\mathcal{F}_{1} \\
& =\epsilon \int_{\partial B} \frac{-g_{1}(X)+B_{1}\left(l^{P}\right)(X)}{\epsilon} \widetilde{u_{0}}(\epsilon X) \mathrm{d} \sigma(X)+\mathcal{F}_{1}+\mathcal{F}_{2} \\
& =\epsilon \int_{\partial B} \frac{-g_{1}(X)+B_{1}\left(l^{P}\right)(X)}{\epsilon} \frac{\epsilon^{2}}{2} \nabla^{2} u_{0}(0) X \cdot X \mathrm{~d} \sigma(X)+\mathcal{F}_{1}+\mathcal{F}_{2}+\mathcal{F}_{3} \\
& =\epsilon^{2} \frac{1}{2} \int_{\partial B} \lambda_{1}(y) \nabla^{2} u_{0}(0) y \cdot y \mathrm{~d} \sigma(y)+\mathcal{F}_{1}+\mathcal{F}_{2}+\mathcal{F}_{3}
\end{aligned}
$$

where

$$
\begin{aligned}
& \mathcal{F}_{1}=\int_{\partial B_{\epsilon}} B_{1}\left(l_{\epsilon}^{e_{\epsilon}}\right) \widetilde{u_{0}}, \quad \mathcal{F}_{2}=\epsilon \int_{\partial B}\left(B_{1}\left(v_{0}\right)(\epsilon X)+\frac{g_{1}(X)}{\epsilon}\right) \widetilde{u_{0}}(\epsilon X) \mathrm{d} \sigma(X), \\
& \mathcal{F}_{3}=\int_{\partial B}\left(-g_{1}(X)+B_{1}\left(l^{P}\right)(X)\right)\left(\widetilde{u_{0}}(\epsilon X)-\epsilon^{2} \frac{1}{2} \nabla^{2} u_{0}(0) X . X\right) \mathrm{d} \sigma(X) .
\end{aligned}
$$


Let $B_{r}$ such as $B \varsubsetneqq B_{r} \subset \frac{1}{\epsilon} \Omega$. By a change of variable, by using Lemma B.2 (Appendix B), a Taylor expansion of $\widetilde{u_{0}}(\epsilon X)$, the trace theorem applied on $B_{r} \backslash \bar{B}$, a change of variable and Lemma B.6 (Appendix B), the following estimate holds

$$
\begin{aligned}
\mathcal{F}_{1} & =\epsilon \int_{\partial B} B_{1}\left(l_{\epsilon}^{e_{\epsilon}}\right)(\epsilon X) \widetilde{u_{0}}(\epsilon X) \mathrm{d} \sigma(X)=\epsilon \int_{\partial B} \frac{1}{\epsilon^{3}} B_{1}\left(l_{\epsilon}^{e_{\epsilon}}(\epsilon X)\right) \widetilde{u_{0}}(\epsilon X) \mathrm{d} \sigma(X) \\
& \leq C\left|l_{\epsilon}^{e_{\epsilon}}(\epsilon X)\right|_{2, B}=C\left|l^{e_{\epsilon}(\epsilon X)}\right|_{2, B} \leq C \|\left. e_{\epsilon}(\epsilon X)\right|_{H^{2}\left(B_{r} \backslash \bar{B}\right) / \mathbb{P}_{1}} \leq C \epsilon\left|e_{\epsilon}\right|_{2, \Omega_{\epsilon}} \leq C \epsilon^{3} \log (\epsilon) .
\end{aligned}
$$

From estimates given in Proposition 3.1, and by a Taylor expansion of $\widetilde{u_{0}}(\epsilon X)$ at 0 we easily see that

$$
\mathcal{F}_{2}=O\left(\epsilon^{3}\right), \quad \mathcal{F}_{3}=O\left(\epsilon^{3}\right) .
$$

Similarly

$$
\begin{aligned}
J_{B} & =\int_{\partial B_{\epsilon}}\left(B_{2}\left(v_{0}\right)+B_{2}\left(l_{\epsilon}^{\epsilon^{2} P\left(\frac{x}{\epsilon}\right)+e_{\epsilon}}\right)\right) \partial_{n} \widetilde{u_{0}}=\epsilon \int_{\partial B}\left(B_{2}\left(v_{0}\right)(\epsilon X)+B_{2}\left(l^{P}\right)(X)\right) \partial_{n} \widetilde{u_{0}}(\epsilon X) \mathrm{d} \sigma(X)+\mathcal{F}_{4}, \\
& =\epsilon \int_{\partial B}\left(-g_{2}(X)+B_{2}\left(l^{P}\right)(X)\right) \partial_{n} \widetilde{u_{0}}(\epsilon X) \mathrm{d} \sigma(X)+\mathcal{F}_{4}+\mathcal{F}_{5}, \\
& =\epsilon^{2} \int_{\partial B}\left(-g_{2}(X)+B_{2}\left(l^{P}\right)(X)\right) \nabla^{2} u_{0}(0) X . n \mathrm{~d} \sigma(X)+\mathcal{F}_{4}+\mathcal{F}_{5}+\mathcal{F}_{6}, \\
& =-\epsilon^{2} \int_{\partial B} \lambda_{2}(y) \nabla^{2} u_{0}(0) y \cdot y \mathrm{~d} \sigma(y)+\mathcal{F}_{4}+\mathcal{F}_{5}+\mathcal{F}_{6},
\end{aligned}
$$

where

$$
\begin{aligned}
& \mathcal{F}_{4}=\int_{\partial B_{\epsilon}} B_{2}\left(l_{\epsilon}^{e_{\epsilon}}\right) \partial_{n} \widetilde{u_{0}}, \quad \mathcal{F}_{5}=\epsilon \int_{\partial B}\left(B_{2}\left(v_{0}\right)(\epsilon X)+g_{2}(X)\right) \partial_{n} \widetilde{u_{0}}(\epsilon X) \mathrm{d} \sigma(X), \\
& \mathcal{F}_{6}=\int_{\partial B}\left(-g_{2}(X)+B_{2}\left(l^{P}\right)(X)\right)\left(\partial_{n} \widetilde{u_{0}}(\epsilon X)-\epsilon^{2} \nabla^{2} u_{0}(0) X . n\right) \mathrm{d} \sigma(X) .
\end{aligned}
$$

Similarly to the $\mathcal{F}_{1}$ computation, and from a Taylor expansion of $\partial_{n} \widetilde{u_{0}}(\epsilon X)$ we can prove that

$$
\mathcal{F}_{4}=O\left(-\epsilon^{3} \log (\epsilon)\right) .
$$

Then from estimates given in Proposition 3.1 and a Taylor expansion of $\partial_{n} \widetilde{u_{0}}(\epsilon X)$ we obtain

$$
\mathcal{F}_{5}=O\left(\epsilon^{3}\right), \quad \mathcal{F}_{6}=O\left(\epsilon^{3}\right) .
$$

Thus, we deduce the estimates of $J_{A}$ and $J_{B}$ given in the lemma.

For $\mathcal{E}_{1}$, by using a change of variable, a Taylor expansion of $\widetilde{u_{0}}(\epsilon X)$ and the definition of $F$ given in (3.10), it is straightforward that

$$
\mathcal{E}_{1}=O\left(\epsilon^{3}\right)
$$

For $\mathcal{E}_{2}$, a change of variable, Lemma B.2 (see Appendix B) with $\epsilon=1$, the trace theorem applied on $B_{r} \backslash \bar{B}$, a change of variable again, and finally Lemma B.6 (see Appendix B) lead to

$$
\begin{aligned}
\mathcal{E}_{2} & =\epsilon^{2} \int_{B} \Delta^{2} u_{0}(\epsilon X) l_{\epsilon}^{w_{\epsilon}}(\epsilon X) \leq C \epsilon^{2}\left\|l^{w_{\epsilon}(\epsilon X)}\right\|_{0, B_{r} \backslash \bar{B}} \leq C \epsilon^{2}\left\|w_{\epsilon}(\epsilon X)\right\|_{2, B}, \\
& \leq C \epsilon^{2}\left(\left\|w_{\epsilon}(\epsilon X)\right\|_{0, B_{r} \backslash \bar{B}}+\left|w_{\epsilon}(\epsilon X)\right|_{1, B_{r} \backslash \bar{B}}+\left|w_{\epsilon}(\epsilon X)\right|_{2, B_{r} \backslash \bar{B}}\right) \\
& \leq C \epsilon^{2}\left(\frac{1}{\epsilon}\left\|w_{\epsilon}\right\|_{0, \Omega_{\epsilon}}+\left|w_{\epsilon}\right|_{1, \Omega_{\epsilon}}+\epsilon\left|w_{\epsilon}\right|_{2, \Omega_{\epsilon}}\right) \leq C \epsilon^{3} \log (\epsilon) .
\end{aligned}
$$

where $B \varsubsetneqq B_{r} \subset \frac{1}{\epsilon} \Omega$. Similarly for $\mathcal{E}_{3}$ we have

$$
\mathcal{E}_{3} \leq C \epsilon\left(\left|w_{\epsilon}\right|_{1, \Omega_{\epsilon}}+\epsilon\left|w_{\epsilon}\right|_{2, \Omega_{\epsilon}}\right) \leq-C \epsilon^{3} \log (\epsilon)
$$




\subsection{Computation of the topological gradient in the case of the ball}

From (3.7), (3.11) and using estimates given in Lemma 3.2 we have

$$
\begin{aligned}
\frac{J_{\epsilon}\left(u_{\epsilon}\right)-J_{0}\left(u_{0}\right)}{\epsilon^{2}}= & \pi\left(f(0)-u_{0}(0)\right) v_{0}(0)+\gamma \pi \nabla u_{0}(0) \cdot \nabla v_{0}(0) \\
& +\frac{1}{2} \int_{\partial B} \lambda_{1}(y) \nabla^{2} u_{0}(0) y \cdot y \mathrm{~d} \sigma(y)+\int_{\partial B} \lambda_{2}(y) \nabla^{2} u_{0}(0) y \cdot y \mathrm{~d} \sigma(y)+\frac{\mathcal{J}_{\epsilon}}{\epsilon^{2}}+o(1) .
\end{aligned}
$$

Using polar coordinates and from the expressions of $\lambda_{1}$ and $\lambda_{2}$ given in (3.17) and (3.18), we obtain

$$
\begin{aligned}
\frac{J_{\epsilon}\left(u_{\epsilon}\right)-J_{0}\left(u_{0}\right)}{\epsilon^{2}}= & \pi\left(f(0)-u_{0}(0)\right) v_{0}(0)+\gamma \pi \nabla u_{0}(0) . \nabla v_{0}(0) \\
& +\left(\frac{\partial^{2} u_{0}}{\partial x_{1}^{2}}(0)-\frac{\partial^{2} u_{0}}{\partial x_{2}^{2}}(0)\right)\left(\frac{\partial^{2} v_{0}}{\partial x_{1}^{2}}(0)-\frac{\partial^{2} v_{0}}{\partial x_{2}^{2}}(0)\right) \pi a^{\prime} \\
& +\frac{\partial^{2} u_{0}}{\partial x_{2} \partial x_{1}}(0) \frac{\partial^{2} v_{0}}{\partial x_{2} \partial x_{1}}(0) \pi 4 a^{\prime}+\pi c^{\prime} \Delta u_{0}(0) \Delta v_{0}(0)+\frac{\mathcal{J}_{\epsilon}}{\epsilon^{2}}+o(1),
\end{aligned}
$$

where

$$
a^{\prime}=-2 \frac{1-\nu}{3+\nu}, \quad c^{\prime}=-\frac{1+\nu}{1-\nu}
$$

From (3.4) and by using Lemma B.6 applied to $u_{\epsilon}-u_{0}$ and a Taylor expansion of $f$ and $u_{0}$ at 0 , we have

$$
\mathcal{J}_{\epsilon}=-\pi \epsilon^{2}\left(f(0)-u_{0}(0)\right) u_{0}(0)+o\left(\epsilon^{2}\right) .
$$

\subsection{Conclusion: general expression for all $x_{0} \in \Omega$}

The topological gradient of the cost function $J_{\epsilon}$ in the case of the ball associated with $\left(\mathcal{P}_{\epsilon}^{b}\right)$ given in $(3.1)$ is for all point $x_{0} \in \Omega$ :

$$
\begin{aligned}
\mathcal{I}\left(x_{0}\right)= & \pi\left(f\left(x_{0}\right)-u_{0}\left(x_{0}\right)\right)\left(v_{0}\left(x_{0}\right)-u_{0}\left(x_{0}\right)\right)+\gamma \pi \nabla u_{0}\left(x_{0}\right) \cdot \nabla v_{0}\left(x_{0}\right) \\
& -\frac{2 \pi(1-\nu)}{3+\nu}\left(\left(\frac{\partial^{2} u_{0}}{\partial x_{1}^{2}}\left(x_{0}\right)-\frac{\partial^{2} u_{0}}{\partial x_{2}^{2}}\left(x_{0}\right)\right)\left(\frac{\partial^{2} v_{0}}{\partial x_{1}^{2}}\left(x_{0}\right)-\frac{\partial^{2} v_{0}}{\partial x_{2}^{2}}\left(x_{0}\right)\right)+4 \frac{\partial^{2} u_{0}}{\partial x_{2} \partial x_{1}}\left(x_{0}\right) \frac{\partial^{2} v_{0}}{\partial x_{2} \partial x_{1}}\left(x_{0}\right)\right) \\
& -\frac{\pi(1+\nu)}{1-\nu} \Delta u_{0}\left(x_{0}\right) \Delta v_{0}\left(x_{0}\right) .
\end{aligned}
$$

\section{STUDY IN THE CASE OF THE CRACK}

\subsection{Notations and statement of the problem}

For each smooth manifold $\Sigma \subset \Omega$, we define the following spaces:

$$
H_{00}^{1 / 2}(\Sigma)=\left\{u_{\mid \Sigma}, u \in H^{1 / 2}(\widetilde{\Sigma}), u_{\mid \widetilde{\Sigma} \backslash \Sigma}=0\right\}, \quad H_{00}^{3 / 2}(\Sigma)=\left\{u_{\mid \Sigma}, u \in H^{3 / 2}(\widetilde{\Sigma}), u_{\mid \widetilde{\Sigma} \backslash \Sigma}=0\right\}
$$

where $\widetilde{\Sigma}$ is a smooth closed manifold containing $\Sigma$ and of the same dimension.

We define on these spaces the following norms

$$
\left\|u_{\mid \Sigma}\right\|_{H_{00}^{1 / 2}(\Sigma)}=\|u\|_{H^{1 / 2}(\widetilde{\Sigma})}, \quad\left\|u_{\mid \Sigma}\right\|_{H_{00}^{3 / 2}(\Sigma)}=\|u\|_{H^{3 / 2}(\widetilde{\Sigma})} .
$$

Now, let $\sigma \subset \Omega$ a $C^{1}$-manifold of dimension 1, with normal $\boldsymbol{n}$ and containing the origin. We denote by $\boldsymbol{\tau}$ the tangent vector to the crack $\sigma$ such as $(\boldsymbol{n}, \boldsymbol{\tau})$ forms an orthonormal basis. $\partial \boldsymbol{\tau}$ denotes the differentiation along the 


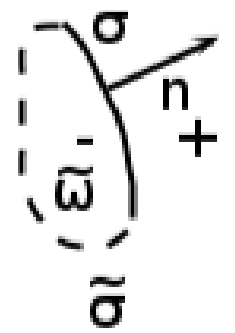

(a) $\widetilde{\sigma} \supset \sigma$ and $\widetilde{\omega}$

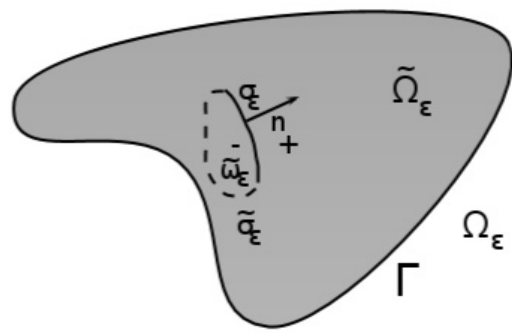

(b) $\sigma_{\epsilon}, \widetilde{\sigma_{\epsilon}}, \widetilde{\omega_{\epsilon}}$ and $\widetilde{\Omega_{\epsilon}}$

FIGURE 2. Extension of the crack in a smooth closed curve

vector $\boldsymbol{\tau}$ and $\mathrm{d} \boldsymbol{\tau}$ stands for the curvilinear Lebesgue measure along $\sigma$. In this section we set the crack exterior domain, $\Lambda=\mathbb{R}^{2} \backslash \bar{\sigma}$. As for the ball we define the following weighted Sobolev space on $\Lambda$ :

$$
W^{2}(\Lambda)=\left\{u, \frac{u}{\left(1+r^{2}\right) \log \left(2+r^{2}\right)} \in L^{2}(\Lambda), \frac{\nabla u}{\left(1+r^{2}\right)^{1 / 2} \log \left(2+r^{2}\right)} \in L^{2}(\Lambda), \nabla^{2} u \in L^{2}(\Lambda)\right\},
$$

where $r=|x|$. We denote by $W^{2}(\Lambda) / \mathbb{P}_{1}$ the space of $W^{2}(\Lambda)$ functions defined up to $\mathbb{P}_{1}$ functions and by $\Omega_{\epsilon}$ the cracked domain $\Omega \backslash \overline{x_{0}+\epsilon \sigma}$. We assume that $\sigma_{\epsilon} \cap \partial \Omega=\emptyset$ and to simplify that $x_{0} \equiv 0$. We set $\sigma_{\epsilon}=\left\{x, \frac{x}{\epsilon} \in \sigma\right\}$. Thus $\partial \Omega_{\epsilon}=\sigma_{\epsilon} \cup \Gamma$ and the problem $\left(\mathcal{P}_{\epsilon}\right)$ expresses as

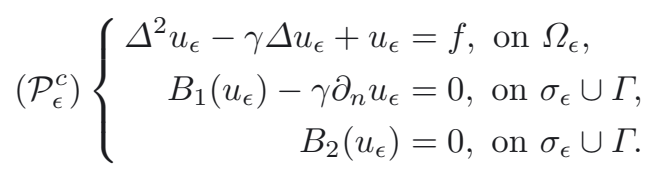

Let $\widetilde{\sigma}$ a smooth closed curve of same dimension as $\sigma$ such as $\sigma \subset \widetilde{\sigma}$, and let $\widetilde{\omega}$ the sub-domain of $\mathbb{R}^{2}$, such as $\partial \widetilde{\omega}=\widetilde{\sigma}$; we denote by $\widetilde{\omega_{\epsilon}}=\left\{x, \frac{x}{\epsilon} \in \widetilde{\omega}\right\}$ and $\widetilde{\Omega_{\epsilon}}=\Omega \backslash \overline{\widetilde{\omega_{\epsilon}}}$ (see Fig. 2). Let $v \in H^{2}\left(\Omega_{\epsilon}\right)$ and $u_{\epsilon}$ the solution of $\left(P_{\epsilon}^{c}\right)$. Thanks to classical regularity result, we obtain that $u_{\epsilon} \in H^{4}\left(\Omega_{\epsilon}\right)$. With an integration by parts in $\Omega \backslash \overline{\bar{\omega}_{\epsilon}} \cup \widetilde{\omega_{\epsilon}}$ (see [9], p. 376) the variational formulation of $\left(\mathcal{P}_{\epsilon}^{c}\right)$ is

$$
\begin{aligned}
\int_{\Omega_{\epsilon}} f v & =\int_{\Omega_{\epsilon}}\left(\Delta^{2} u_{\epsilon}-\gamma \Delta u_{\epsilon}+u_{\epsilon}\right) v \\
& =a_{\epsilon}(u, v)-\int_{\sigma_{\epsilon}}\left(\left(B_{1}\left(u_{\epsilon}\right)-\gamma \partial_{n} u_{\epsilon}\right)[v]-B_{2}\left(u_{\epsilon}\right)\left[\partial_{n} v\right]\right)+\int_{\Gamma}\left(B_{1}\left(u_{\epsilon}\right) v-B_{2}\left(u_{\epsilon}\right) \partial_{n} v\right), \forall v \in H^{2}\left(\Omega_{\epsilon}\right),
\end{aligned}
$$

where $a_{\epsilon}(u, v)$ is given in $(2.3)$ and where we set $[v]=v^{+}-v^{-}$the jump of $v$ through $\sigma_{\epsilon}$ and $\left[\partial_{n} v\right]=$ $\left(\partial_{n} v\right)^{+}-\left(\partial_{n} v\right)^{-}$the jump of $\partial_{n} v$ (see Fig. 2). To simplify, we assume that $\sigma=\{(s, 0),-1<s<1\}$ (we place us in the local coordinate system of the crack).

We compute the topological gradient as in the case of the ball in evaluating the leading term in the asymptotic expansion of $J_{\epsilon}\left(u_{\epsilon}\right)-J_{0}\left(u_{0}\right)$ when $\epsilon \rightarrow 0$. By using equations that $u_{\epsilon}$ and $u_{0}$ verify, we have

$$
J_{\epsilon}\left(u_{\epsilon}\right)-J_{0}\left(u_{0}\right)=\int_{\Omega_{\epsilon}}\left(f-2 u_{0}\right)\left(u_{\epsilon}-u_{0}\right)-\int_{\Omega_{\epsilon}}\left(u_{\epsilon}-u_{0}\right)^{2} .
$$

We define $L_{\epsilon}(u)$ as in (3.3), and we set

$$
\mathcal{J}_{\epsilon}=-\int_{\Omega_{\epsilon}}\left(u_{\epsilon}-u_{0}\right)^{2} .
$$


As in [2] and [17], to evaluate (4.2), we introduce $v_{\epsilon}$ the solution of the adjoint problem (3.5). From (3.5) and (4.3) we rewrite $(4.2)$

$$
\begin{aligned}
J_{\epsilon}\left(u_{\epsilon}\right)-J_{0}\left(u_{0}\right) & =-a_{\epsilon}\left(u_{\epsilon}-u_{0}, v_{\epsilon}\right)+\mathcal{J}_{\epsilon}=-l_{\epsilon}\left(v_{\epsilon}\right)+a_{\epsilon}\left(u_{0}, v_{\epsilon}\right)+\mathcal{J}_{\epsilon} \\
& =-\int_{\Omega_{\epsilon}} f v_{\epsilon}+\int_{\Omega_{\epsilon}}\left(\Delta^{2} u_{0}-\gamma \Delta u_{0}+u_{0}\right) v_{\epsilon}+\int_{\sigma_{\epsilon}} B_{1}\left(u_{0}\right)\left[v_{\epsilon}\right]-B_{2}\left(u_{0}\right)\left[\partial_{n} v_{\epsilon}\right]+\mathcal{J}_{\epsilon} \\
& =\int_{\sigma_{\epsilon}}\left(B_{1}\left(u_{0}\right)-\gamma \partial_{n} u_{0}\right)\left[v_{\epsilon}\right]-B_{2}\left(u_{0}\right)\left[\partial_{n} v_{\epsilon}\right]+\mathcal{J}_{\epsilon} .
\end{aligned}
$$

Then we set $w_{\epsilon}=v_{\epsilon}-v_{0}$, where $v_{0}$ is solution of (3.5), with $\epsilon=0$. Thus, $J_{\epsilon}\left(u_{\epsilon}\right)-J_{0}\left(u_{0}\right)$ rewrites as

$$
J_{\epsilon}\left(u_{\epsilon}\right)-J_{0}\left(u_{0}\right)=\int_{\sigma_{\epsilon}}\left(B_{1}\left(u_{0}\right)-\gamma \partial_{n} u_{0}\right)\left[w_{\epsilon}\right]-B_{2}\left(u_{0}\right)\left[\partial_{n} w_{\epsilon}\right]+\mathcal{J}_{\epsilon}=I_{A}-I_{B}+\mathcal{J}_{\epsilon},
$$

where

$$
I_{A}=\int_{\sigma_{\epsilon}}\left(B_{1}\left(u_{0}\right)-\gamma \partial_{n} u_{0}\right)\left[w_{\epsilon}\right], \quad I_{B}=\int_{\sigma_{\epsilon}} B_{2}\left(u_{0}\right)\left[\partial_{n} w_{\epsilon}\right] .
$$

In the next subsection, we show that $I_{A}$ and $I_{B} \backsim O\left(\epsilon^{2}\right)$ and that $\mathcal{J}_{\epsilon} \backsim o\left(\epsilon^{2}\right)$. We first establish the asymptotic expansion of $B_{1}\left(v_{0}\right), B_{2}\left(v_{0}\right)$ and $w_{\epsilon}$.

\subsection{Estimates of $B_{1}\left(v_{0}\right)(x)$ and $B_{2}\left(v_{0}\right)(x)$ for $x \in \sigma_{\epsilon}$}

Proposition 4.1. By assuming that $v_{0}$ is smooth, and by setting $X=(s, 0)$ for $-1<s<1$, we have the following boundary expansions when $\epsilon \rightarrow 0$

$$
B_{1}\left(v_{0}\right)(\epsilon X)=O(1), \quad B_{2}\left(v_{0}\right)(\epsilon X)=-g_{2}(X)+O(\epsilon),
$$

where

$$
g_{2}(X)=-\frac{\partial^{2} v_{0}}{\partial x_{2}^{2}}(0)-\nu \frac{\partial^{2} v_{0}}{\partial x_{1}^{2}}(0)
$$

Proof. It is straightforward by using that $\partial \tau=-\partial x_{1}$ and $\partial n=\partial x_{2}$.

\subsection{Asymptotic expansion of $w_{\epsilon}$}

We recall that $w_{\epsilon}=v_{\epsilon}-v_{0}$ is the solution of

$$
\left(\mathcal{Q}_{\epsilon}^{c}\right)\left\{\begin{aligned}
\Delta^{2} w_{\epsilon}-\gamma \Delta w_{\epsilon}+w_{\epsilon} & =0, \text { on } \Omega_{\epsilon} \\
B_{1}\left(w_{\epsilon}\right)-\gamma \partial_{n} w_{\epsilon} & =-B_{1}\left(v_{0}\right)+\gamma \partial_{n} v_{0}, \text { on } \sigma_{\epsilon}, \\
B_{2}\left(w_{\epsilon}\right) & =-B_{2}\left(v_{0}\right), \text { on } \sigma_{\epsilon}, \\
B_{1}\left(w_{\epsilon}\right)-\gamma \partial_{n} w_{\epsilon} & =B_{2}\left(w_{\epsilon}\right)=0, \text { on } \Gamma .
\end{aligned}\right.
$$

To estimate $w_{\epsilon}$, we introduce the following exterior problem

$$
\left(\mathcal{R}_{\text {ext }}\right)\left\{\begin{aligned}
\Delta^{2} R & =0, \text { on } \mathbb{R}^{2} \backslash \bar{\sigma}, \\
B_{1}(R) & =g_{1}, \text { on } \sigma \\
B_{2}(R) & =g_{2}, \text { on } \sigma .
\end{aligned}\right.
$$

We easily verify that $g_{1} \in\left(H_{00}^{3 / 2}(\sigma)\right)^{\prime}$ and $g_{2} \in\left(H_{00}^{1 / 2}(\sigma)\right)^{\prime}$. Using Theorem C.3 given in Appendix C, we deduce that problem $\left(\mathcal{R}_{\text {ext }}\right)$ admits a unique solution $R \in W^{2}(\Lambda) / \mathbb{P}_{1}$ which is a sum of third and fourth layers potential

$$
R(x)=\oint_{\sigma} \lambda_{1}(y) B_{1, y}(E(x-y)) \mathrm{d} \tau(y)+\oint_{\sigma} \lambda_{2}(y) B_{2, y}(E(x-y)) \mathrm{d} \tau(y),
$$


where $\oint$ is the principal Cauchy value. We have the following relations:

$$
[R]=R_{\mid \sigma}^{+}-R_{\mid \sigma}^{-}=\lambda_{1}, \quad\left[\partial_{n} R\right]=\partial_{n} R_{\mid \sigma}^{+}-\partial_{n} R_{\mid \sigma}^{-}=-\lambda_{2},
$$

where

$$
\begin{aligned}
\lambda_{1}(s) & =0, \quad \lambda_{2}(s)=\frac{-4 \beta}{(1-\nu)(3+\nu)} \sqrt{1-s^{2}}, \quad \forall(s, 0) \in \sigma, \\
\beta & =\frac{\partial^{2} v_{0}}{\partial x_{2}^{2}}(0)+\nu \frac{\partial^{2} v_{0}}{\partial x_{1}^{2}}(0) .
\end{aligned}
$$

By using Lemma C.5 (Appendix C), we obtain

$$
w_{\epsilon}=\epsilon^{2} R\left(\frac{x}{\epsilon}\right)+e_{\epsilon}
$$

with $\left\|e_{\epsilon}\right\|_{H^{2}\left(\Omega_{\epsilon}\right)}=O\left(-\epsilon^{2} \log (\epsilon)\right)$. In the next subsection we show that $I_{A} \backsim o\left(\epsilon^{2}\right)$ and that $I_{B} \backsim O\left(\epsilon^{2}\right)$.

\subsection{Estimates of $I_{A}$ and $I_{B}$}

Lemma 4.2. Let $I_{A}$ and $I_{B}$ given in (4.5), then we have the following estimates

$$
I_{A}=O\left(\epsilon^{3}\right), \quad I_{B}=-\epsilon^{2} \xi \int_{\sigma} \lambda_{2}(X) \mathrm{d} \tau(X)+O\left(\epsilon^{3}\right)
$$

with $\xi=\frac{\partial^{2} u_{0}}{\partial x_{2}^{2}}(0)+\nu \frac{\partial^{2} u_{0}}{\partial x_{1}^{2}}(0)$.

Proof. We assume that $u_{0}$ is smooth, thus from Proposition 4.1 we have

$$
B_{1}\left(u_{0}\right)(\epsilon X)=O(1) \text {. }
$$

Let $B \supset \sigma$, from Lemma C.1 (see Appendix C), and by using the equivalency of the $H^{2}(B \backslash \bar{\sigma})$-norm and the seminorm, with a change of variable, we get:

$$
I_{A} \leq \epsilon\left\|B_{1}\left(u_{0}\right)(\epsilon X)\right\|_{H_{00}^{3 / 2}(\sigma)^{\prime}}\left\|w_{\epsilon}(\epsilon X)\right\|_{H^{2}(B \backslash \bar{\sigma}) / \mathbb{P}_{1}} \leq C \epsilon\left|w_{\epsilon}(\epsilon X)\right|_{2, B \backslash \bar{\sigma}} \leq C \epsilon^{2}\left|w_{\epsilon}\right|_{2, \Omega_{\epsilon}} .
$$

From Lemma C.5 (see Appendix C), we have

$$
I_{A}=O\left(\epsilon^{3}\right)
$$

From (4.8), (4.10) and (4.5), we express $I_{B}$ as

$$
I_{B}=-\epsilon^{2} \xi \int_{\sigma} \lambda_{2}(X) \mathrm{d} \tau(X)+\mathcal{E}_{1}+\mathcal{E}_{2},
$$

where

$$
\begin{aligned}
\mathcal{E}_{1} & =\epsilon \int_{\sigma}\left(B_{2}\left(u_{0}\right)(\epsilon X)-\xi\right)\left[\partial_{n} w_{\epsilon}(\epsilon X)\right] \mathrm{d} \tau(X), \quad \mathcal{E}_{2}=\epsilon \int_{\sigma} \xi\left[\partial_{n} e_{\epsilon}(\epsilon X)\right] \mathrm{d} \tau(X), \\
\xi & =\frac{\partial^{2} u_{0}}{\partial x_{2}^{2}}(0)+\nu \frac{\partial^{2} u_{0}}{\partial x_{1}^{2}}(0)
\end{aligned}
$$

Similarly by using Proposition 4.1 and Lemma C.5 we get

$$
\begin{aligned}
& \mathcal{E}_{1} \leq\left\|B_{2}\left(u_{0}\right)(\epsilon X)-\xi\right\|_{H_{00}^{3 / 2}(\sigma)^{\prime}}\left\|w_{\epsilon}(\epsilon X)\right\|_{H^{2}(B \backslash \bar{\sigma}) / \mathbb{P}_{1}} \leq C \epsilon^{2}\left|w_{\epsilon}\right|_{2, \Omega_{\epsilon}} \leq C \epsilon^{3}, \\
& \mathcal{E}_{2} \leq C\left\|e_{\epsilon}(\epsilon X)\right\|_{H^{2}(B \backslash \bar{\sigma}) / \mathbb{P}_{1}} \leq C \epsilon\left|e_{\epsilon}\right|_{2, \Omega_{\epsilon}} \leq C \epsilon^{3} \log (\epsilon) .
\end{aligned}
$$




\subsection{Computation of the topological gradient in the case of the crack}

From (4.2), and from estimates given in Lemma 4.2 we have

$$
\frac{J_{\epsilon}\left(u_{\epsilon}\right)-J_{0}\left(u_{0}\right)}{\epsilon^{2}}=\xi \int_{\sigma} \lambda_{2}(X) \mathrm{d} \tau(X)+\frac{\mathcal{J}_{\epsilon}}{\epsilon^{2}}+o(1)
$$

By Lemma C.5 applied to $u_{\epsilon}-u_{0}$ we deduce the estimate of $\mathcal{I}_{\epsilon}$ :

$$
\mathcal{I}_{\epsilon}=O\left(\epsilon^{4}(\log (\epsilon))^{2}\right) \text {. }
$$

By using the expression of $\lambda_{2}$ given in (4.9), we deduce the topological gradient at 0

$$
\mathcal{I}(0)=\xi \int_{\sigma} \lambda_{2}(X) \mathrm{d} \tau(X)=\xi \int_{-1}^{1} \frac{-4 \beta}{(1-\nu)(3+\nu)} \sqrt{1-s^{2}} \mathrm{~d} s=\frac{-2 \pi \xi \beta}{(1-\nu)(3+\nu)}
$$

or more precisely

$$
\mathcal{I}\left(0, \boldsymbol{e}_{\boldsymbol{y}}\right)=-\frac{2 \pi}{(1-\nu)(3+\nu)}\left(\frac{\partial^{2} u_{0}}{\partial x_{2}^{2}}(0)+\nu \frac{\partial^{2} u_{0}}{\partial x_{1}^{2}}(0)\right)\left(\frac{\partial^{2} v_{0}}{\partial x_{2}^{2}}(0)+\nu \frac{\partial^{2} v_{0}}{\partial x_{1}^{2}}(0)\right) .
$$

\subsection{Conclusion: general expression for all point $x_{0} \in \Omega$}

The topological gradient expression associated with the cost function $J_{\epsilon}(u)$ and problem $\left(\mathcal{P}_{\epsilon}^{c}\right)$ given in $(3.1)$ and in the case of a cracked domain $\Omega_{\epsilon}=\Omega \backslash \overline{x_{0}+\epsilon \sigma(\boldsymbol{n})}$ is:

$$
\mathcal{I}\left(x_{0}, \boldsymbol{n}\right)=-\frac{2 \pi}{(1-\nu)(3+\nu)}\left(\nabla^{2} u_{0}\left(x_{0}\right)(\boldsymbol{n}, \boldsymbol{n})+\nu \nabla^{2} u_{0}\left(x_{0}\right)(\boldsymbol{\tau}, \boldsymbol{\tau})\right)\left(\nabla^{2} v_{0}\left(x_{0}\right)(\boldsymbol{n}, \boldsymbol{n})+\nu \nabla^{2} v_{0}\left(x_{0}\right)(\boldsymbol{\tau}, \boldsymbol{\tau})\right),
$$

where $\boldsymbol{n} \perp \boldsymbol{\tau}$ such that $(\boldsymbol{\tau}, \boldsymbol{n})$ be an orthonormal basis. Then, if we want to minimize $J_{\epsilon}\left(u_{\epsilon}\right)$ we can define the topological gradient at $x_{0}$ as the min value of $\mathcal{I}\left(x_{0}, \boldsymbol{n}\right)$ (this definition should change according to the application: see [10] for another definition adapted to a fine structure detection problem):

$$
\mathcal{I}\left(x_{0}\right)=\min _{\|\boldsymbol{n}\|=1} \mathcal{I}\left(x_{0}, \boldsymbol{n}\right) .
$$

\section{Appendix A}

In this Appendix we give a useful result for the study of exterior problems. We denote by the same letter $\mathcal{O}$ the exterior domain of the crack and of the ball. In the following, we denote by $B_{r}$ the ball of center 0 and of radius $r$. In the crack case, $B$ denotes a ball containing strictly the crack (for example $B=B_{\eta}$ with $\eta>1$ ) while in the ball's one $B$ denotes the unit ball. We denote by $W^{2}(\mathcal{O})$ the weighted Sobolev space defined by (3.14) replacing $B^{\prime}$ by $\mathcal{O}$.

Lemma A.1. Let $u \in W^{2}(\mathcal{O})$. We have the following inequality

$$
\|u\|_{W^{2}(\mathcal{O}) / \mathbb{P}_{1}} \leq C|u|_{2, \mathcal{O}}
$$

where $C$ is a constant depending only on $\mathcal{O}$.

Proof. Let $\eta>0$ such as $B=B_{\eta}: 2>\eta>1$ for the crack and $\eta=1$ for the ball. Let $\varphi \in C^{2}([0,+\infty[)$, a real function defined by:

$$
\left\{\begin{array}{lr}
\varphi=0, & \text { for } 0 \leq t \leq \eta \\
0 \leq \varphi \leq 1 & \text { for } \eta \leq t \leq 2 \\
\varphi=1 & \text { for } t \geq 2
\end{array}\right.
$$


Let $\psi(x)=\varphi(|x|)$, then $u \psi \in W_{0}^{2}\left(B^{\prime}\right)$, where we recall that $W_{0}^{2}\left(B^{\prime}\right)$ is the closure in $W^{2}\left(B^{\prime}\right)$ (see $(3.14)$ ) of $C^{\infty}\left(B^{\prime}\right)$-functions with compact support in $B^{\prime}$.

On $W_{0}^{2}\left(B^{\prime}\right)$, thanks to Hardy inequality and to the $\psi$ expression, we have

$$
\|u\|_{W^{2}\left(B_{2}^{\prime}\right)} \leq\|u \psi\|_{W_{0}^{2}\left(B^{\prime}\right)} \leq C|\psi u|_{2, B^{\prime}}
$$

Now we bound from above $|\psi u|_{2, B^{\prime}}$ :

$$
|\psi u|_{2, B^{\prime}} \leq|u|_{2, B_{2}^{\prime}}+|\psi u|_{2, B_{2} \backslash \bar{B}} \leq|u|_{2, B_{2}^{\prime}}+\|\psi\|_{2, B_{2} \backslash \bar{B}}\|u\|_{2, B_{2} \backslash \bar{B}} \leq|u|_{2, B_{2}^{\prime}}+C\|u\|_{2, B_{2} \backslash \bar{B}} .
$$

Denoting by $\omega$ the crack or the unit ball and $\mathcal{O}=\mathbb{R}^{2} \backslash \bar{\omega}$, and using the equivalency of the $W^{2}\left(B_{2} \backslash \bar{\omega}\right)$-norm with the $H^{2}\left(B_{2} \backslash \bar{\omega}\right)$-norm we get

$$
\|u\|_{W^{2}(\mathcal{O})} \leq C\|u\|_{2, B_{2} \backslash \bar{\omega}}+C|u|_{2, B_{2}^{\prime}} .
$$

Thanks to Deny-Lions Lemma ([9], Lem. 5.2) we get

$$
\|u\|_{W^{2}(\mathcal{O}) / \mathbb{P}_{1}} \leq C\|u\|_{H^{2}\left(B_{2} \backslash \bar{\omega}\right) / \mathbb{P}_{1}}+C|u|_{2, B_{2}^{\prime}} \leq C|u|_{2, \mathcal{O}}
$$

\section{Appendix B}

In this section we develop some technical computations not given in Section 3. We recall that $B$ denotes the unit ball and $B^{\prime}$ the exterior domain. We recall the definition of the weighted Sobolev space $W^{2}\left(B^{\prime}\right)$ :

$$
W^{2}\left(B^{\prime}\right)=\left\{u, \frac{u}{\left(1+r^{2}\right) \log \left(2+r^{2}\right)} \in L^{2}\left(B^{\prime}\right), \frac{\nabla u}{\left(1+r^{2}\right)^{1 / 2} \log \left(2+r^{2}\right)} \in L^{2}\left(B^{\prime}\right), \nabla^{2} u \in L^{2}\left(B^{\prime}\right)\right\}
$$

with $r=|x|$.

Lemma B.1. Let $u \in H^{2}(\Omega)$, such as $\Delta^{2} u \in L^{2}(\Omega)$. Let $\Gamma_{1}$ a smooth closed curve such as $\Gamma_{1} \subset \partial \Omega$. We have the following inequality

$$
\left(\begin{array}{l}
B_{1}(u) \\
B_{2}(u)
\end{array}\right)_{H^{-3 / 2}\left(\Gamma_{1}\right) \times H^{-1 / 2}\left(\Gamma_{1}\right)} \leq C|u|_{2, \Omega}+\left\|\Delta^{2} u\right\|_{0, \Omega}
$$

where

$$
\left(\begin{array}{l}
B_{1}(u) \\
B_{2}(u)
\end{array}\right)_{H^{-3 / 2}\left(\Gamma_{1}\right) \times H^{-1 / 2}\left(\Gamma_{1}\right)}=\sup _{\left\|\varphi_{1}\right\|_{3 / 2, \Gamma_{1}}+\left\|\varphi_{2}\right\|_{1 / 2, \Gamma_{1}}=1} \int_{\Gamma_{1}} B_{1}(u) \varphi_{1}-B_{2}(u) \varphi_{2} .
$$

Proof. See [11].

Lemma B.2. Let $\omega \subset \mathbb{R}^{2}, \varphi_{1} \in H^{3 / 2}(\partial \omega)$ and $\varphi_{2} \in H^{1 / 2}(\partial \omega)$, then there is a unique solution $l^{\varphi_{1}, \varphi_{2}} \in H^{2}(\omega)$ of ( see (3.6) with $\epsilon=1)$ :

$$
\left\{\begin{aligned}
\Delta^{2} l^{\varphi_{1}, \varphi_{2}} & =0, \text { on } \omega \\
l^{\varphi_{1}, \varphi_{2}} & =\varphi_{1}, \text { on } \partial \omega \\
\partial_{n} l^{\varphi_{1}, \varphi_{2}} & =\varphi_{2}, \text { on } \partial \omega
\end{aligned}\right.
$$

and we have the following inequality

$$
\left\|l^{\varphi_{1}, \varphi_{2}}\right\|_{2, \omega} \leq C\left\|\varphi_{1}\right\|_{3 / 2, \partial \omega}+C\left\|\varphi_{2}\right\|_{1 / 2, \partial \omega} .
$$

Proof. See [11]. 
The four first points of the following theorem are taken from ([9], p. 402 and p. 417). Let $f \in H^{s}(\partial B)$ and $g \in H^{-s}(\partial B)$, we denote by $\langle f, g\rangle$ the scalar product on $\partial B$ :

$$
\langle f, g\rangle=\int_{\partial B} f g \mathrm{~d} \tau
$$

Theorem B.3. Let $g_{1} \in H^{-3 / 2}(\partial B), g_{2} \in H^{-1 / 2}(\partial B)$, such as

$$
\left\langle g_{1}, 1\right\rangle=0, \quad\left\langle g_{1}, x_{1}\right\rangle-\left\langle g_{2}, n_{1}\right\rangle=0, \quad\left\langle g_{1}, x_{2}\right\rangle-\left\langle g_{2}, n_{2}\right\rangle=0
$$

We consider the following exterior problem

$$
\left(\mathcal{P}_{\text {ext }}\right)\left\{\begin{array}{c}
\Delta^{2} P=0, \text { on } B^{\prime} \\
B_{1}(P)=g_{1}, \text { on } \partial B, \\
B_{2}(P)=g_{2}, \text { on } \partial B .
\end{array}\right.
$$

(1) The problem $\left(\mathcal{P}_{\text {ext }}\right)$ given in (B.3) admits a unique solution in $W^{2}\left(B^{\prime}\right) / \mathbb{P}_{1}$ and the map $\left(g_{1}, g_{2}\right) \longmapsto P$ is continuous from $H^{-3 / 2}(\partial B) \times H^{-1 / 2}(\partial B)$ to $W^{2}\left(B^{\prime}\right) / \mathbb{P}_{1}$, where $W^{2}\left(B^{\prime}\right) / \mathbb{P}_{1}$ is the space of $W^{2}\left(B^{\prime}\right)$ functions defined up to a polynomial function of degree less or equal than 1.

(2) The solution $P \in W^{2}\left(B^{\prime}\right) / \mathbb{P}_{1}$ of (B.3) expresses as the sum of simple and double layers potential:

$$
P(x)=\int_{\partial B} \lambda_{1}(y) E(x-y) \mathrm{d} \sigma(y)+\int_{\partial B} \lambda_{2}(y) \partial_{n_{y}} E(x-y) \mathrm{d} \sigma(y), \text { for } x \in B^{\prime} .
$$

(3) There exist $a_{0}, a_{1}, a_{2} \in \mathbb{R}$ such as the densities $\lambda_{1}$ and $\lambda_{2}$ are given by the five following equations

$$
\begin{array}{r}
-\frac{1}{2} \lambda_{1}(x)+\int_{\partial B} \lambda_{1}(y) B_{1, x}(E(x-y)) \mathrm{d} \sigma(y)+\oint_{\partial B} \lambda_{2}(y) B_{1, x}\left(\partial_{n y} E(x-y)\right) \mathrm{d} \sigma(y)+a_{0}+a_{1} x_{1}+a_{2} x_{2}=g_{1}(x) \\
+\frac{1}{2} \lambda_{2}(x)+\int_{\partial B} \lambda_{1}(y) B_{2, x}(E(x-y)) \mathrm{d} \sigma(y)+\int_{\partial B} \lambda_{2}(y) B_{2, x}\left(\partial_{n y} E(x-y)\right) \mathrm{d} \sigma(y)-a_{1} n_{1}(x)-a_{2} n_{2}(x)=g_{2}(x) \\
\left\langle\lambda_{1}, 1\right\rangle=0 \\
\left\langle\lambda_{1}, x_{1}\right\rangle+\left\langle\lambda_{2}, n_{1}\right\rangle=0 \\
\left\langle\lambda_{1}, x_{2}\right\rangle+\left\langle\lambda_{2}, n_{2}\right\rangle=0
\end{array}
$$

where $x \in \partial B$ and $\oint$ denotes the principal Cauchy value.

The three last conditions of (B.5) express the following asymptotic behavior of P:

$$
P(x)=a_{0}+a_{1} x_{1}+a_{2} x_{2}+A \log (|x|)+O(1) .
$$

(4) For $x \in \partial B$, we set $x=(\cos (\theta), \sin (\theta))$ for $\theta \in\left[0,2 \pi\left[\right.\right.$. Assuming that $g_{1}$ and $g_{2}$ take the form $g_{1}(x)=A_{1} \cos (2 \theta)+B_{1} \sin (2 \theta)$, and $g_{2}(x)=C_{2}+A_{2} \cos (2 \theta)+B_{2} \sin (2 \theta)$, then $P(x)=O(\log (|x|))$ and then $a_{0}=a_{1}=a_{2}=0$. Thus $\lambda_{1}$ and $\lambda_{2}$ are given by:

$$
\begin{aligned}
& \lambda_{1}(x)=\alpha \cos (2 \theta)+\beta \sin (2 \theta) \\
& \lambda_{2}(x)=c+a \cos (2 \theta)+b \sin (2 \theta)
\end{aligned}
$$

with

$$
\begin{array}{ll}
\alpha=-8 \frac{A_{1}+A_{2}(1+\nu)}{(1-\nu)(3+\nu)}, & \beta=-8 \frac{B_{1}+B_{2}(1+\nu)}{(1-\nu)(3+\nu)} \\
a=2 \frac{A_{1}(1+\nu)+4 A_{2}}{(1-\nu)(3+\nu)}, & b=2 \frac{B_{1}(1+\nu)+4 B_{2}}{(1-\nu)(3+\nu)}, \quad c=2 \frac{C_{2}}{1-\nu}
\end{array}
$$


(5) Let $l^{P}$ defined by (3.6) $($ for $\epsilon=1)$ and the data $g_{1}$ and $g_{2}$ given in the fourth point; then we have the following jump relations through $\partial B$ for $x \in \partial B$

$$
\begin{aligned}
& g_{1}(x)-B_{1}\left(l^{P}\right)(x)=-\lambda_{1}(x), x \in \partial B \\
& g_{2}(x)-B_{2}\left(l^{P}\right)(x)=\lambda_{2}(x), x \in \partial B
\end{aligned}
$$

Remark B.4. The choice of $g_{1}$ and $g_{2}$ in the fourth point comes from the boundaries data given in Proposition 3.1.

Proof. We introduce the bilinear form on $W^{2}\left(B^{\prime}\right) / \mathbb{P}_{1}$

$$
b^{\prime}(u, v)=\int_{B^{\prime}} \Delta u \Delta v+(1-\nu)\left(2 \frac{\partial^{2} u}{\partial x_{1} \partial x_{2}} \frac{\partial^{2} v}{\partial x_{1} \partial x_{2}}-\frac{\partial^{2} u}{\partial x_{1}^{2}} \frac{\partial^{2} v}{\partial x_{2}^{2}}-\frac{\partial^{2} u}{\partial x_{2}^{2}} \frac{\partial^{2} v}{\partial x_{1}^{2}}\right), \forall u, v \in W^{2}\left(B^{\prime}\right) / \mathbb{P}_{1}
$$

and the linear form on $W^{2}\left(B^{\prime}\right) / \mathbb{P}_{1}$

$$
l^{\prime}(v)=\int_{\partial B} g_{1} v-g_{2} \partial_{n} v, \forall v \in W^{2}\left(B^{\prime}\right) / \mathbb{P}_{1}
$$

From (2.2), and Lemma A.1, the coercivity of $b^{\prime}(u, v)$ is straightforward. Applying the trace theorem on $B_{2} \backslash \bar{B}$, we deduce the continuity of $l^{\prime}(v)$ on $W^{2}\left(B^{\prime}\right) / \mathbb{P}_{1}$, which ends the proof of the first point.

The second and the third point are proven in ([9], p. 417).

Then by using the Green formula on $B^{\prime}$, we get

$$
\begin{aligned}
P(x)= & \int_{B^{\prime}}-\Delta^{2} E(x-y) P(y) \mathrm{d} \sigma_{y} \\
= & \int_{\partial B} B_{1, y}(E(x-y)) P(y) \mathrm{d} \sigma_{y}-\int_{\partial B} B_{2, y}(E(x-y)) \partial_{n} P(y) \mathrm{d} \sigma_{y}, \\
& +\int_{\partial B} \partial_{n_{y}}(E(x-y)) B_{2, y}(P)(y) \mathrm{d} \sigma_{y}-\int_{\partial B} E(x-y) B_{1, y}(P)(y) \mathrm{d} \sigma_{y} . \\
= & A-B+C-D
\end{aligned}
$$

We remark that with the values of $g_{1}$ and $g_{2}$ given in the fourth point we have the following relations:

$$
\begin{aligned}
\left\langle g_{1}, 1\right\rangle & =0 \\
\left\langle g_{1}, x_{1}\right\rangle & =\left\langle g_{1}, n_{1}\right\rangle=\left\langle g_{1}, x_{2}\right\rangle=\left\langle g_{1}, n_{2}\right\rangle=0, \\
\left\langle g_{2}, x_{1}\right\rangle & =\left\langle g_{2}, n_{1}\right\rangle=\left\langle g_{2}, x_{2}\right\rangle=\left\langle g_{2}, n_{2}\right\rangle=0 .
\end{aligned}
$$

Computing $B_{1, y}(E(x-y))$ and $B_{2, y}(E(x-y))$ for $y \in \partial B$ and when $|x| \rightarrow \infty$ we show that

$$
A=\int_{\partial B} B_{1, y}(E(x-y)) P(y) \mathrm{d} y=O(\log (|x|)), \quad B=\int_{\partial B} B_{2, y}(E(x-y)) \partial_{n} P(y) \mathrm{d} y=O(\log (|x|)) .
$$

For $C$ and $D$, we use a Taylor expansion of $E(x-y)$ and $\partial_{n_{y}}(E(x-y))$ at 0 , and we assume that $|x| \rightarrow \infty$

$$
\begin{aligned}
E(x-y) & =E(x)-y \cdot \nabla E(x)+\frac{1}{2} y \cdot \nabla^{2} E(x) y+O\left(\frac{1}{|x|}\right), \\
\partial_{n_{y}} E(x-y) & =\nabla E(x) \cdot n_{y}-\nabla^{2} E(x) y \cdot n_{y}+O\left(\frac{1}{|x|}\right) .
\end{aligned}
$$


Using the estimates $\nabla^{2} E(x)=O(\log (|x|))$, (B.9) and the relations (B.8) we have

$$
\begin{aligned}
& C=\int_{\partial B} \partial_{n_{y}}(E(x-y)) B_{2, y}(P)(y) \mathrm{d} y=O(\log (|x|)) \\
& D=\int_{\partial B} E(x-y) B_{1, y}(P)(y) \mathrm{d} y=O(\log (|x|))
\end{aligned}
$$

We deduce that $P(x)=O(\log (|x|))$ when $x \rightarrow \infty$ and then $a_{0}=a_{1}=a_{2}=0$.

To determinate $\lambda_{1}$ and $\lambda_{2}$ we first compute kernels associated with the integral equations.

From ([9], pp. 390-392), by setting $x=(\cos (\varphi), \sin (\varphi))$ and $y=(\cos (\theta), \sin (\theta))$, we have

$$
\begin{aligned}
B_{2, x}(E(x-y)) & =-\frac{1}{8 \pi}((1+\nu) \log (2(1-\cos (\varphi-\theta))+(1+3 \nu)+(1-\nu)(1-\cos (\varphi-\theta))), \\
B_{2, x}\left(\partial_{n_{y}}(E(x-y))\right) & =-\frac{\nu}{4 \pi}+\frac{1-\nu}{8 \pi} \cos (\varphi-\theta), \\
B_{1, x}(E(x-y)) & =-\frac{1}{4 \pi}-\frac{1-\nu}{8 \pi} \cos (\varphi-\theta), \\
B_{1, x}\left(\partial_{n_{y}}(E(x-y))\right) & =-\frac{1-\nu}{8 \pi} \cos (\varphi-\theta)+\frac{1+\nu}{8 \pi(1-\cos (\varphi-\theta))} .
\end{aligned}
$$

To simplify notations, we denote by $\lambda_{1}(\theta)=\lambda_{1}(\cos (\theta), \sin (\theta))$ and $\lambda_{2}(\theta)=\lambda_{2}(\cos (\theta), \sin (\theta))$ the densities $\lambda_{1}$ and $\lambda_{2}$ evaluated on the unit circle. Equations given in the third point of the Theorem B.3 express as

(Eq. 1) $\left\{\begin{array}{l}-\frac{1}{2} \lambda_{1}(\varphi)+\int_{0}^{2 \pi}\left(-\frac{1}{4 \pi}-\frac{1-\nu}{8 \pi} \cos (\varphi-\theta)\right) \lambda_{1}(\theta) \mathrm{d} \theta \\ +\oint_{0}^{2 \pi}\left(-\frac{1-\nu}{8 \pi} \cos (\varphi-\theta)+\frac{1+\nu}{8 \pi(1-\cos (\varphi-\theta))}\right) \lambda_{2}(\theta) \mathrm{d} \theta=A_{1} \cos (2 \varphi)+B_{1} \sin (2 \varphi),\end{array}\right.$
(Eq. 2) $\left\{\begin{array}{l}\frac{1}{2} \lambda_{2}(\varphi)+\int_{0}^{2 \pi}\left(-\frac{1}{8 \pi}((1+\nu) \log (2(1-\cos (\varphi-\theta))+(1+3 \nu)+(1-\nu)(1-\cos (\varphi-\theta)))) \lambda_{1}(\theta) \mathrm{d} \theta\right. \\ +\int_{0}^{2 \pi}\left(-\frac{\nu}{4 \pi}+\frac{1-\nu}{8 \pi} \cos (\varphi-\theta)\right) \lambda_{2}(\theta) \mathrm{d} \theta=C_{2}+A_{2} \cos (2 \varphi)+B_{2} \sin (2 \varphi) .\end{array}\right.$

The only singular kernel is $\frac{1+\nu}{8 \pi(1-\cos (\varphi-\theta))}=O\left(|\theta-\varphi|^{2}\right)$ associated with $B_{1, x}\left(\partial_{n_{y}}(E(x-y))\right)$. We remark that $B_{1, x}\left(\partial_{n_{y}}(E(x-y))\right)=\partial_{n_{x}}\left(B_{1, y}(E(x-y))\right)$. We deduce that

$$
\int_{\partial B} B_{1, x}\left(\partial_{n_{y}}(E(x-y))\right) \mathrm{d} \sigma(y)=\partial_{n_{x}} \int_{\partial B} B_{1, y}(E(x-y)) \mathrm{d} \sigma(y)=\partial_{n_{x}}\left(-\frac{1}{2}\right)=0 .
$$

We can rewrite the left term of (Eq. 1) as:

$$
\oint_{0}^{2 \pi}\left(-\frac{1-\nu}{8 \pi} \cos (\varphi-\theta)+\frac{1+\nu}{8 \pi(1-\cos (\varphi-\theta))}\right)\left(\lambda_{2}(\theta)-\lambda_{2}(\varphi)\right) d \theta
$$

Then we are searching for the form of the solutions. First $\int_{0}^{2 \pi} \lambda_{1}(\theta) \mathrm{d} \theta=0$ and according to the fourth point, $g_{1} \in \operatorname{span}(\cos (2 \theta), \sin (2 \theta))$ and $g_{2} \in \operatorname{span}(1, \cos (2 \theta), \sin (2 \theta))$ so regarding the kernels forms, it is legitimate to search $\lambda_{1}$ and $\lambda_{2}$ as

$$
\lambda_{1}(\theta)=\alpha \cos (2 \theta)+\beta \sin (2 \theta), \quad \lambda_{2}(\theta)=a \cos (2 \theta)+b \sin (2 \theta)+c .
$$

Let $f$ continuous on $[0,2 \pi]$, we define the following improper integral: $I(f)(\varphi)=\oint_{0}^{2 \pi} \frac{f(\theta)-f(\varphi)}{1-\cos (\theta-\varphi)} \mathrm{d} \theta$, where by definition: $\oint_{0}^{2 \pi}=\lim _{\epsilon \rightarrow 0} \int_{0}^{\varphi-\epsilon}+\int_{\varphi+\epsilon}^{2 \pi}$. Then we compute the two following improper integrals:

$$
I(\cos (2 \theta))(\varphi)=-4 \pi \cos (2 \varphi), \quad I(\sin (2 \theta))(\varphi)=-4 \pi \sin (2 \varphi) .
$$


We rewrite (Eq. 1) as

$$
\begin{aligned}
& -\frac{1}{2}(\alpha \cos (2 \varphi)+\beta \sin (2 \varphi))+\int_{0}^{2 \pi}\left(-\frac{1}{4 \pi}-\frac{1-\nu}{8 \pi} \cos (\varphi-\theta)\right)(\alpha \cos (2 \theta)+\beta \sin (2 \theta)) \mathrm{d} \theta \\
& +\oint_{0}^{2 \pi}\left(-\frac{1-\nu}{8 \pi} \cos (\varphi-\theta)+\frac{1+\nu}{8 \pi(1-\cos (\varphi-\theta))}\right)(a(\cos (2 \theta)-\cos (2 \varphi))+b(\sin (2 \theta)-\sin (2 \varphi))) \mathrm{d} \theta \\
& =A_{1} \cos (2 \varphi)+B_{1} \sin (2 \varphi) .
\end{aligned}
$$

From (B.10),we obtain the following equation:

$$
-\frac{1}{2}(\alpha \cos (2 \varphi)+\beta \sin (2 \varphi))+\frac{1+\nu}{8 \pi}(-4 \pi a \cos (2 \varphi)-4 \pi b \sin (2 \varphi))=A_{1} \cos (2 \varphi)+B_{1} \sin (2 \varphi) .
$$

Identifying each term in front of cosinus and sinus we get

$$
(\widetilde{\text { Eq. 1 }})\left\{\begin{array}{l}
-\frac{1+\nu}{2} a-\frac{\alpha}{2}=A_{1}, \\
-\frac{1+\nu}{2} b-\frac{\beta}{2}=B_{1}
\end{array}\right.
$$

Similarly, equation (Eq. 2) rewrites as

$$
(\widetilde{\text { Eq. } 2})\left\{\begin{aligned}
\frac{1-\nu}{2} c & =C_{2}, \\
\frac{a}{2}+\frac{1+\nu}{8} \alpha & =A_{2}, \\
\frac{b}{2}+\frac{1+\nu}{8} \beta & =B_{2} .
\end{aligned}\right.
$$

Solving $(\widetilde{\text { Eq. } 1})$ and $(\widetilde{\text { Eq. } 2})$ we obtain the expression of $\lambda_{1}$ and $\lambda_{2}$ given in (B.6), which ends the proof of the fourth point.

For the fifth point, let $l^{P}$ defined by $(3.6)$ (with $\epsilon=1$ ). Thanks to the continuity of simple and double layers (see [9], p. 384), we have

$$
l^{P}(x)=\int_{\partial B} \lambda_{1}(y) E(x-y) \mathrm{d} \sigma(y)+\int_{\partial B} \lambda_{2}(y) \partial_{n_{y}} E(x-y) \mathrm{d} \sigma(y), \text { for } x \in B .
$$

Using again jump relations given in ([9], p. 385), we obtain for $x \in \partial B$

$$
\begin{aligned}
& B_{1}\left(l^{P}\right)(x)=\frac{1}{2} \lambda_{1}(x)+\int_{\partial B} \lambda_{1}(y) B_{1, x}(E(x-y)) \mathrm{d} \sigma(y)+\int_{\partial B} \lambda_{2}(y) B_{1, x}\left(\partial_{n_{y}} E(x-y)\right) \mathrm{d} \sigma(y), \\
& B_{2}\left(l^{P}\right)(x)=-\frac{1}{2} \lambda_{2}(x)+\int_{\partial B} \lambda_{1}(y) B_{2, x}(E(x-y)) \mathrm{d} \sigma(y)+\int_{\partial B} \lambda_{2}(y) B_{2, x}\left(\partial_{n_{y}} E(x-y)\right) \mathrm{d} \sigma(y) .
\end{aligned}
$$

Finally, by using (B.13) and the two first equations of (B.5) and by setting $a_{0}=a_{1}=a_{2}=0$, we get the last point of the theorem.

Lemma B.5. Let $P$ the solution of $\left(\mathcal{P}_{\text {ext }}\right)$ given in (3.15). We have the following asymptotic behavior as $|x| \rightarrow \infty$ :

$$
\begin{gathered}
|P(x)| \leq C \log (|x|), \quad|\nabla P(x)| \leq \frac{C}{|x|}, \quad\left|\nabla^{2} P(x)\right| \leq \frac{C}{|x|^{2}} \\
\left\|P\left(\frac{x}{\epsilon}\right)\right\|_{0, \Omega_{\epsilon}}=O(-\log (\epsilon)), \quad\left|P\left(\frac{x}{\epsilon}\right)\right|_{1, \Omega_{\epsilon}}=O(\sqrt{-\log (\epsilon)}), \quad\left|P\left(\frac{x}{\epsilon}\right)\right|_{2, \Omega_{\epsilon}}=O\left(\frac{1}{\epsilon}\right)
\end{gathered}
$$


Proof. $P(x)$ is given by

$$
P(x)=\int_{\partial B} \lambda_{1}(y) E(x-y) \mathrm{d} \sigma(y)+\int_{\partial B} \lambda_{2}(y) \partial_{n_{y}}(E(x-y)) \mathrm{d} \sigma(y)
$$

With the boundary data $g_{1}$ and $g_{2}$ given in the last point of Theorem B.3, $\lambda_{1}$ and $\lambda_{2}$ verify the following relations

$$
\begin{aligned}
\left\langle\lambda_{1}, 1\right\rangle & =0, \\
\left\langle\lambda_{1}, x_{1}\right\rangle & =\left\langle\lambda_{1}, n_{1}\right\rangle=\left\langle\lambda_{1}, x_{2}\right\rangle=\left\langle\lambda_{1}, n_{2}\right\rangle=0, \\
\left\langle\lambda_{2}, x_{1}\right\rangle & =\left\langle\lambda_{2}, n_{1}\right\rangle=\left\langle\lambda_{2}, x_{2}\right\rangle=\left\langle\lambda_{2}, n_{2}\right\rangle=0 .
\end{aligned}
$$

A Taylor expansion of $P$ at $x$ and (B.14) give the first inequality. The two others inequalities are straightforward if we differentiate the expression of $P$ in interchanging integral and derivatives. Then using again a Taylor expansion at $x$, and by taking $|x| \longrightarrow \infty$, we get the result. To estimate the $L^{2}\left(\Omega_{\epsilon}\right)$-norms of the derivatives of $P$, we choose two large positive reals a and $\mathrm{D}$ and a small positive real $\epsilon$ such that $B \subset B_{a} \subset \frac{1}{\epsilon} \Omega \subset \frac{1}{\epsilon} B_{D}$. Thus we have:

$$
\begin{aligned}
\left\|P\left(\frac{x}{\epsilon}\right)\right\|_{0, \Omega_{\epsilon}}^{2} & =\int_{\Omega_{\epsilon}} P\left(\frac{x}{\epsilon}\right)^{2} \mathrm{~d} x=\epsilon^{2} \int_{\frac{1}{\epsilon} \Omega \backslash \bar{B}} P(y)^{2} \mathrm{~d} y \leq \epsilon^{2}\left(\int_{B_{a} \backslash \bar{B}} P(y)^{2} \mathrm{~d} y+\int_{\frac{1}{\epsilon} B_{D} \backslash \overline{B_{a}}} P(y)^{2} \mathrm{~d} y\right) \\
& \leq C \epsilon^{2}+C \epsilon^{2} \int_{a}^{D / \epsilon} \log (r)^{2} r \mathrm{~d} r=C \epsilon^{2}+C \epsilon^{2}\left[\log (r) \frac{r^{2}}{2}(\log (r)-1)+\frac{r^{2}}{4}\right]_{a}^{\frac{D}{\epsilon}} \leq C \log (\epsilon)^{2}
\end{aligned}
$$

which is the first estimate. The computation of the second and the third norm estimate are similar.

Lemma B.6. Let $w_{\epsilon}$ given in (3.13) and $P$ the solution of $\left(\mathcal{P}_{\text {ext }}\right)$ the exterior problem given in (3.15), then we have the following asymptotic expansion when $\epsilon \rightarrow 0$ :

$$
w_{\epsilon}=\epsilon^{2} P\left(\frac{x}{\epsilon}\right)+e_{\epsilon}
$$

with

$$
\begin{array}{r}
\left\|e_{\epsilon}\right\|_{2, \Omega_{\epsilon}}=O\left(\epsilon^{2} \log (\epsilon)\right), \quad\left\|w_{\epsilon}\right\|_{0, \Omega_{\epsilon}}=O\left(-\epsilon^{2} \log (\epsilon)\right) \\
\left|w_{\epsilon}\right|_{1, \Omega_{\epsilon}}=O\left(-\epsilon^{2} \log (\epsilon)\right), \quad\left|w_{\epsilon}\right|_{2, \Omega_{\epsilon}}=O(\epsilon)
\end{array}
$$

Proof. $e_{\epsilon}$ is solution of the following problem

$$
\left(\mathcal{E}_{\epsilon}\right)\left\{\begin{aligned}
\Delta^{2} e_{\epsilon}-\alpha \Delta e_{\epsilon}+e_{\epsilon} & =\alpha \Delta P\left(\frac{x}{\epsilon}\right)-\epsilon^{2} P\left(\frac{x}{\epsilon}\right) & & \text { on } \Omega_{\epsilon} \\
B_{1}\left(e_{\epsilon}\right)-\gamma \partial_{n} e_{\epsilon} & =-B_{1}\left(v_{0}\right)-\frac{1}{\epsilon} g_{1}\left(\frac{x}{\epsilon}\right)-\gamma \partial_{n} v_{0}=\varphi_{1}(x)=O(1), & & \text { on } \partial B_{\epsilon} \\
B_{2}\left(e_{\epsilon}\right) & =-B_{2}\left(v_{0}\right)-g_{2}\left(\frac{x}{\epsilon}\right)=\varphi_{2}(x)=O(x), & & \text { on } \partial B_{\epsilon} \\
B_{1}\left(e_{\epsilon}\right)-\gamma \partial_{n} e_{\epsilon} & =-\frac{1}{\epsilon} B_{1}(P)\left(\frac{x}{\epsilon}\right)=\phi_{1}(x)=O\left(\frac{\epsilon^{2}}{|x|^{3}}\right), & & \text { on } \Gamma \\
B_{2}\left(e_{\epsilon}\right) & =-B_{2}(P)\left(\frac{x}{\epsilon}\right)=\phi_{2}(x)=O\left(\frac{\epsilon^{2}}{|x|^{2}}\right), & & \text { on } \Gamma
\end{aligned}\right.
$$

where the expressions of $g_{1}$ and $g_{2}$ are:

$$
g_{1}(X)=A_{1} \cos (2 \theta)+B_{1} \sin (2 \theta), \quad g_{2}(X)=C_{2}+A_{2} \cos (2 \theta)+B_{2} \sin (2 \theta)
$$


with $X=(\cos (\theta), \sin (\theta))$ and where

$$
\begin{array}{ll}
A_{1}=(1-\nu)\left(\frac{\partial^{2} v_{0}}{\partial x_{1}^{2}}(0)-\frac{\partial^{2} v_{0}}{\partial x_{1}^{2}}(0)\right), & B_{1}=2(1-\nu) \frac{\partial^{2} v_{0}}{\partial x_{1} \partial x_{2}}(0), \\
A_{2}=-\frac{1-\nu}{2}\left(\frac{\partial^{2} v_{0}}{\partial x_{1}^{2}}(0)-\frac{\partial^{2} v_{0}}{\partial x_{2}^{2}}(0)\right), \quad B_{2}=-(1-\nu) \frac{\partial^{2} v_{0}}{\partial x_{1} \partial x_{2}}(0), \quad C_{2}=-\frac{1+\nu}{2} \Delta v_{0}(0) .
\end{array}
$$

The variational formulation of problem (B.15) is: find $e_{\epsilon} \in H^{2}\left(\Omega_{\epsilon}\right)$ such as

$$
a_{\epsilon}\left(e_{\epsilon}, v\right)=\int_{\Omega_{\epsilon}}\left(-\epsilon^{2} P\left(\frac{x}{\epsilon}\right)+\gamma \Delta P\left(\frac{x}{\epsilon}\right)\right) v+\int_{\partial B_{\epsilon}} \varphi_{1} v-\varphi_{2} \partial_{n} v+\int_{\Gamma}-\phi_{1} v+\phi_{2} \partial_{n} v .
$$

We integrate by parts the second terms in the right hand side part of the above equality

$$
\int_{\Omega_{\epsilon}} \Delta P\left(\frac{x}{\epsilon}\right) v=\int_{\Gamma} \epsilon \partial_{n} P\left(\frac{x}{\epsilon}\right) v-\epsilon^{2} P\left(\frac{x}{\epsilon}\right) \partial_{n} v-\int_{\partial B_{\epsilon}} \epsilon \partial_{n} P\left(\frac{x}{\epsilon}\right) v+\epsilon^{2} P\left(\frac{x}{\epsilon}\right) \partial_{n} v+\int_{\Omega_{\epsilon}} \epsilon^{2} P\left(\frac{x}{\epsilon}\right) \Delta v .
$$

Let $b_{\epsilon}$ the bilinear form associated with the leading operator of (B.15) such as for all $u, v \in H^{2}\left(\Omega_{\epsilon}\right)$

$$
b_{\epsilon}(u, v)=\int_{\Omega_{\epsilon}} \Delta u \Delta v+(1-\nu)\left(2 \frac{\partial^{2} u}{\partial x_{1} \partial x_{2}} \frac{\partial^{2} v}{\partial x_{1} \partial x_{2}}-\frac{\partial^{2} u}{\partial x_{1}^{2}} \frac{\partial^{2} v}{\partial x_{2}^{2}}-\frac{\partial^{2} u}{\partial x_{2}^{2}} \frac{\partial^{2} v}{\partial x_{1}^{2}}\right) .
$$

Then we split the error into the sum $e_{\epsilon}=e_{\epsilon}^{1}+e_{\epsilon}^{2}$, where

- $e_{\epsilon}^{1} \in H^{2}\left(\Omega_{\epsilon}\right) / \mathbb{P}_{1}$ is defined by

$$
b_{\epsilon}\left(e_{\epsilon}^{1}, v\right)=l_{\epsilon}^{1}(v), \forall v \in H^{2}\left(\Omega_{\epsilon}\right) / \mathbb{P}_{1}
$$

with

$$
l_{\epsilon}^{1}(v)=\int_{\partial B_{\epsilon}}\left(\varphi_{1}-\epsilon \partial_{n} P\left(\frac{x}{\epsilon}\right)\right) v+\int_{\partial B_{\epsilon}}\left(-\varphi_{2}+\epsilon^{2} P\left(\frac{x}{\epsilon}\right)\right) \partial_{n} v
$$

We can show (as for the Poincaré inequality (see [16])), that there exists $C$ independent of $\epsilon$ such as the following inequality holds:

$$
\|u\|_{H^{2}\left(\Omega_{\epsilon}\right) / \mathbb{P}_{1}} \leq C|u|_{2, \Omega_{\epsilon}}, \forall u \in H^{2}\left(\Omega_{\epsilon}\right) / \mathbb{P}_{1} .
$$

We deduce the coercivity of $b_{\epsilon}$ on this space with a constant not depending on $\epsilon$ :

$$
b_{\epsilon}(u, u) \geq(1-\nu)|u|_{2, \Omega_{\epsilon}}^{2} \geq C\|u\|_{H^{2}\left(\Omega_{\epsilon}\right) / \mathbb{P}_{1}}^{2} .
$$

Thanks to the trace theorem applied on $\Omega_{\epsilon}$, the continuity of $l_{\epsilon}^{1}$ on $H^{2}\left(\Omega_{\epsilon}\right) / \mathbb{P}_{1}$ is then straightforward.

- $e_{\epsilon}^{2} \in H^{2}\left(\Omega_{\epsilon}\right)$ is defined by

$$
a_{\epsilon}\left(e_{\epsilon}^{2}, v\right)=l_{\epsilon}^{2}(v), \forall v \in H^{2}\left(\Omega_{\epsilon}\right)
$$

with

$$
\begin{aligned}
l_{\epsilon}^{2}(v)= & \int_{\Omega_{\epsilon}}\left(-\epsilon^{2} P\left(\frac{x}{\epsilon}\right)+\gamma \Delta e_{\epsilon}^{1}-e_{\epsilon}^{1}\right) v+\gamma \epsilon^{2} P\left(\frac{x}{\epsilon}\right) \Delta v \\
& +\int_{\Gamma}\left(-\phi_{1}+\gamma \epsilon \partial_{n} P\left(\frac{x}{\epsilon}\right)\right) v+\left(\phi_{2}-\gamma \epsilon^{2} P\left(\frac{x}{\epsilon}\right)\right) \partial_{n} v .
\end{aligned}
$$

The coercivity of $a_{\epsilon}$ on $H^{2}\left(\Omega_{\epsilon}\right)$ is known (see (2.2)) and as for $b_{\epsilon}$ the constant do not depend on $\epsilon$; the continuity of $l_{\epsilon}^{2}$ on $H^{2}\left(\Omega_{\epsilon}\right)$ is easily checked using the trace theorem on $\Omega_{\epsilon}$. 
Now let us estimate $\left\|e_{\epsilon}^{1}\right\|_{H^{2}\left(\Omega_{\epsilon}\right) / \mathbb{P}_{1}}$ and $\left\|e_{\epsilon}^{2}\right\|_{2, \Omega_{\epsilon}}$ with respect to $\epsilon$.

By considering the variational form of $e_{\epsilon}^{1}$, by taking as test function $v=e_{\epsilon}^{1}$ and by using a change of variable we have

$$
\begin{aligned}
(1-\nu)\left|e_{\epsilon}^{1}\right|_{2, \Omega_{\epsilon}}^{2} \leq b_{\epsilon}\left(e_{\epsilon}^{1}, e_{\epsilon}^{1}\right)= & \epsilon \int_{\partial B}\left(\varphi_{1}(\epsilon X)-\epsilon \partial_{n} P(X)\right) e_{\epsilon}^{1}(\epsilon X)+\epsilon \int_{\partial B}\left(-\varphi_{2}(\epsilon X)+\epsilon^{2} P(X)\right) \partial_{n} e_{\epsilon}^{1}(\epsilon X) \\
\leq & \left(\epsilon\left\|\varphi_{1}(\epsilon X)\right\|_{-3 / 2, \partial B}+\epsilon^{2}\left\|\partial_{n} P\right\|_{-3 / 2, \partial B}\right)\left\|e_{\epsilon}^{1}(\epsilon X)\right\|_{H^{3 / 2}(\partial B) / \mathbb{P}_{1}} \\
& +\left(\left\|\varphi_{2}(\epsilon X)\right\|_{-1 / 2, \partial B}+\epsilon^{2}\|P\|_{-1 / 2, \partial B}\right)\left\|\partial_{n}\left(e_{\epsilon}^{1}(\epsilon X)\right)\right\|_{H^{1 / 2}(\partial B) / \mathbb{P}_{1}} .
\end{aligned}
$$

Let $r>0$ such as $B \nsubseteq B_{r} \subset \frac{1}{\epsilon} \Omega$. The trace theorem on $B_{r} \backslash \bar{B}$, the Deny-Lions inequality ([9], Lem. 5.2) and a change of variable give:

$$
\begin{aligned}
\left|e_{\epsilon}^{1}\right|_{2, \Omega_{\epsilon}}^{2} \leq & C\left(\epsilon\left\|\varphi_{1}(\epsilon X)\right\|_{-3 / 2, \partial B}+\epsilon^{2}\left\|\partial_{n} P\right\|_{-3 / 2, \partial B}\right)\left\|e_{\epsilon}^{1}(\epsilon X)\right\|_{H^{2}\left(B_{r} \backslash \bar{B}\right) / \mathbb{P}_{1}} \\
& +C\left(\left\|\varphi_{2}(\epsilon X)\right\|_{-1 / 2, \partial B}+\epsilon^{2}\|P\|_{-1 / 2, \partial B}\right)\left\|e_{\epsilon}^{1}(\epsilon X)\right\|_{H^{2}\left(B_{r} \backslash \bar{B}\right) / \mathbb{P}_{1}} \\
\leq & C\left(\epsilon\left\|\varphi_{1}(\epsilon X)\right\|_{-3 / 2, \partial B}+\epsilon^{2}\left\|\partial_{n} P\right\|_{-3 / 2, \partial B}\right)\left|e_{\epsilon}^{1}(\epsilon X)\right|_{2, B_{r} \backslash \bar{B}} \\
& +C\left(\left\|\varphi_{2}(\epsilon X)\right\|_{-1 / 2, \partial B}+\epsilon^{2}\|P\|_{-1 / 2, \partial B}\right)\left|e_{\epsilon}^{1}(\epsilon X)\right|_{2, B_{r} \backslash \bar{B}} \\
\leq & C \epsilon\left(\epsilon\left\|\varphi_{1}(\epsilon X)\right\|_{-3 / 2, \partial B}+\epsilon^{2}\left\|\partial_{n} P\right\|_{-3 / 2, \partial B}\right)\left|e_{\epsilon}^{1}\right|_{2, \Omega_{\epsilon}}+C \epsilon\left(\left\|\varphi_{2}(\epsilon X)\right\|_{-1 / 2, \partial B}+\epsilon^{2}\|P\|_{-1 / 2, \partial B}\right)\left|e_{\epsilon}^{1}\right|_{2, \Omega_{\epsilon}} .
\end{aligned}
$$

Using that $\varphi_{1}(\epsilon X)=O(1)$ and $\varphi_{2}(\epsilon X)=O(\epsilon)$ we get

$$
\left\|e_{\epsilon}^{1}\right\|_{H^{2}\left(\Omega_{\epsilon}\right) / \mathbb{P}_{1}}=O\left(\epsilon^{2}\right)
$$

With a similar reasoning for $e_{\epsilon}^{2}$, from its variational formulation we obtain

$$
\begin{aligned}
C\left\|e_{\epsilon}^{2}\right\|_{2, \Omega_{\epsilon}}^{2} \leq a_{\epsilon}\left(e_{\epsilon}^{2}, e_{\epsilon}^{2}\right)= & \int_{\Omega_{\epsilon}}\left(-\epsilon^{2} P\left(\frac{x}{\epsilon}\right)+\gamma \Delta e_{\epsilon}^{1}-e_{\epsilon}^{1}\right) e_{\epsilon}^{2}+\gamma \epsilon^{2} P\left(\frac{x}{\epsilon}\right) \Delta e_{\epsilon}^{2} \\
& +\int_{\Gamma}\left(-\phi_{1}+\gamma \epsilon \partial_{n} P\left(\frac{x}{\epsilon}\right)\right) e_{\epsilon}^{2}+\left(\phi_{2}-\gamma \epsilon^{2} P\left(\frac{x}{\epsilon}\right)\right) \partial_{n} e_{\epsilon}^{2} \\
\leq & C\left(\epsilon^{2}\left\|P\left(\frac{x}{\epsilon}\right)\right\|_{0, \Omega_{\epsilon}}+\gamma\left|e_{\epsilon}^{1}\right|_{2, \Omega_{\epsilon}}+\left\|e_{\epsilon}^{1}\right\|_{L^{2}\left(\Omega_{\epsilon}\right) / \mathbb{P}_{1}}\right)\left\|e_{\epsilon}^{2}\right\|_{0, \Omega_{\epsilon}}+C \gamma \epsilon^{2}\left\|P\left(\frac{x}{\epsilon}\right)\right\|_{0, \Omega_{\epsilon}}\left|e_{\epsilon}^{2}\right|_{2, \Omega_{\epsilon}} \\
& +\left(\left\|\phi_{1}\right\|_{-3 / 2, \Gamma}+\gamma \epsilon\left\|\partial_{n} P\left(\frac{x}{\epsilon}\right)\right\|_{-3 / 2, \Gamma}\right)\left\|e_{\epsilon}^{2}\right\|_{3 / 2, \Gamma} \\
& +\left(\left\|\phi_{2}\right\|_{-1 / 2, \Gamma}+\gamma \epsilon^{2}\left\|P\left(\frac{x}{\epsilon}\right)\right\|_{-1 / 2, \Gamma}\right)\left\|e_{\epsilon}^{2}\right\|_{1 / 2, \Gamma}
\end{aligned}
$$

The trace theorem applied on $\Omega \backslash \overline{B_{r}}$, the estimates $\phi_{1}=O\left(\frac{\epsilon^{2}}{|x|^{3}}\right), \phi_{2}=O\left(\frac{\epsilon^{2}}{|x|^{2}}\right)$ and $\left\|e_{\epsilon}^{1}\right\|_{H^{2}\left(\Omega_{\epsilon}\right) / \mathbb{P}_{1}}=O\left(\epsilon^{2}\right)$, and Lemma B.5 give:

$$
\left\|e_{\epsilon}^{2}\right\|_{2, \Omega_{\epsilon}}=O\left(-\epsilon^{2} \log (\epsilon)\right)
$$

Coming back to $e_{\epsilon}$ since $e_{\epsilon}^{1}$ is defined up to a function in $\mathbb{P}_{1}$, and thanks to the Deny-Lions inequality given in (B.17), we have

$$
\left\|e_{\epsilon}\right\|_{2, \Omega_{\epsilon}} \leq\left\|e_{\epsilon}^{1}\right\|_{H^{2}\left(\Omega_{\epsilon}\right) / \mathbb{P}_{1}}+\left\|e_{\epsilon}^{2}\right\|_{2, \Omega_{\epsilon}} \leq C \epsilon^{2} \log (\epsilon)
$$

which is the first estimate. For the $L^{2}\left(\Omega_{\epsilon}\right)$-norm estimates of $w_{\epsilon}, \nabla w_{\epsilon}$ and $\nabla^{2} w_{\epsilon}$, we must use that $w_{\epsilon}=$ $\epsilon^{2} P\left(\frac{x}{\epsilon}\right)+e_{\epsilon}$, differentiate it, take the norm and use Lemma B.5 and the previous estimate of $\left\|e_{\epsilon}\right\|_{2, \Omega_{\epsilon}}$. 


\section{Appendix C}

Let $\sigma$ a smooth manifold that we extend to a smooth closed curve $\widetilde{\sigma}$. We denote by $\widetilde{\omega}$ the domain such as $\partial \widetilde{\omega}=\widetilde{\sigma}$ (see Fig. 2a).

We recall the definitions of $H_{00}^{1 / 2}(\sigma)$ and $H_{00}^{3 / 2}(\sigma)$ :

$$
H_{00}^{1 / 2}(\sigma)=\left\{u_{\mid \sigma}, u \in H^{1 / 2}(\widetilde{\sigma}), u_{\mid \widetilde{\sigma} \backslash \bar{\sigma}}=0\right\}, \quad H_{00}^{3 / 2}(\sigma)=\left\{u_{\mid \sigma}, u \in H^{3 / 2}(\widetilde{\sigma}), u_{\mid \widetilde{\sigma} \backslash \bar{\sigma}}=0\right\},
$$

and we introduce the weighted Sobolev space

$$
W^{2}(\Lambda)=\left\{u, \frac{u}{\left(1+r^{2}\right) \log \left(2+r^{2}\right)} \in L^{2}(\Lambda), \frac{\nabla u}{\left(1+r^{2}\right)^{1 / 2} \log \left(2+r^{2}\right)} \in L^{2}(\Lambda), \nabla^{2} u \in L^{2}(\Lambda)\right\},
$$

where $r=|x|$ and $\Lambda=\mathbb{R}^{2} \backslash \bar{\sigma}$.

In the sequel, $B$ denotes the ball containing strictly the crack $\sigma$ and its extension $\widetilde{\sigma}$ (for example $B=B_{\eta}$ with $\eta>1$ ) and such as $B \subset \frac{\Omega}{\epsilon}$ for small $\epsilon$.

Lemma C.1. Let $v \in H^{2}(B \backslash \bar{\sigma}), g_{1} \in\left(H_{00}^{3 / 2}(\sigma)\right)^{\prime}$ and $g_{2} \in\left(H_{00}^{1 / 2}(\sigma)\right)^{\prime}$, then we have the following inequality

$$
\int_{\sigma} g_{1}[v] \leq C\left\|g_{1}\right\|_{H_{00}^{3 / 2}(\sigma)^{\prime}}\|v\|_{H^{2}(B \backslash \bar{\sigma}) / \mathbb{P}_{1}}, \quad \int_{\sigma} g_{2}\left[\partial_{n} v\right] \leq C\left\|g_{2}\right\|_{H_{00}^{1 / 2}(\sigma)^{\prime}}\|v\|_{H^{2}(B \backslash \bar{\sigma}) / \mathbb{P}_{1}} .
$$

Proof. By using the definition of the $H_{00}^{3 / 2}(\sigma)$-norm and by splitting the jump of $v$ across $\widetilde{\sigma}$, we have for any smooth function $\psi$ :

$$
\int_{\sigma} g_{1}[v] \leq\left\|g_{1}\right\|_{H_{00}^{3 / 2}(\sigma)^{\prime}}\|[v+\psi]\|_{H_{00}^{3 / 2}(\sigma)} \leq\left\|g_{1}\right\|_{H_{00}^{3 / 2}(\sigma)^{\prime}}\left(\left\|v^{+}+\psi\right\|_{3 / 2, \widetilde{\sigma}}+\left\|v^{-}+\psi\right\|_{3 / 2, \tilde{\sigma})} .\right.
$$

Then by using the trace theorem applied to $\left(v^{+}+\psi\right)$ on $B \backslash \overline{\widetilde{\omega}}$ and $v^{-}+\psi$ on $\widetilde{\omega}$, we have

$$
\int_{\sigma} g_{1}[v] \leq\left\|g_{1}\right\|_{H_{00}^{3 / 2}(\sigma)^{\prime}}\left(\|v+\psi\|_{2, B \backslash \bar{\omega}}+\|v+\psi\|_{2, \tilde{\omega}}\right) .
$$

By taking the infimum with respect to $\psi \in \mathbb{P}_{1}$ and from the Deny-Lions inequality the first inequality holds. The second one is proved in the same manner.

Lemma C.2. Let $u \in H^{2}\left(\Delta^{2}, B \backslash \bar{\sigma}\right), h_{1} \in H_{00}^{3 / 2}(\sigma)$ and $h_{2} \in H_{00}^{1 / 2}(\sigma)$, then we have the following inequality

$$
\int_{\sigma} B_{1}(u) q_{1}-\int_{\sigma} B_{2}(u) q_{2} \leq C\left(\left\|q_{1}\right\|_{H_{00}^{3 / 2}(\sigma)}+\|q\|_{H_{00}^{1 / 2}(\sigma)}\right)\left(|u|_{2, B \backslash \bar{\sigma}}+\left\|\Delta^{2} u\right\|_{L^{2}(B \backslash \bar{\sigma})}\right)
$$

Proof. See [11].

Then we introduce the exterior problem:

$$
\left(\mathcal{R}_{\text {ext }}\right)\left\{\begin{aligned}
\Delta^{2} R & =0, \text { on } \mathbb{R}^{2} \backslash \bar{\sigma}, \\
B_{1}(R) & =g_{1}, \text { on } \sigma, \\
B_{2}(R) & =g_{2}, \text { on } \sigma,
\end{aligned}\right.
$$

where $g_{1} \in H_{00}^{3 / 2}(\sigma)^{\prime}$ and $g_{2} \in H_{00}^{1 / 2}(\sigma)^{\prime}$. 


\section{Theorem C.3.}

(1) (C.1) admits a unique solution in $W^{2}(\Lambda) / \mathbb{P}_{1}$ and the map $\left(g_{1}, g_{2}\right) \longmapsto R$ from $H_{00}^{3 / 2}(\sigma)^{\prime} \times H_{00}^{3 / 2}(\sigma)^{\prime}$ into $W^{2}(\Lambda) / \mathbb{P}_{1}$ is continuous.

(2) The solution of (C.1) expresses as the sum of double and triple layers potential:

$$
R(x)=\int_{\sigma} \lambda_{1}(y) B_{1}(E(x-y)) \mathrm{d} \tau(y)+\int_{\sigma} \lambda_{2}(y) B_{2}(E(x-y)) \mathrm{d} \tau(y), \text { for } x \in \Lambda .
$$

(3) The following jump relations through $\sigma$ hold:

$$
\begin{aligned}
{[R] } & =R_{\mid \sigma}^{+}-R_{\mid \sigma}^{-}=\lambda_{1}, \\
{\left[\partial_{n} R\right] } & =\partial_{n} R_{\mid \sigma}^{+}-\partial_{n} R_{\mid \sigma}^{-}=-\lambda_{2} .
\end{aligned}
$$

(4) The densities $\lambda_{1}$ and $\lambda_{2}$ are given by the two following equations:

$$
\begin{aligned}
& g_{1}(x)=\oint_{\sigma} \lambda_{1}(y) B_{1, x} B_{1, y}(E(x-y)) \mathrm{d} \tau(y)+\oint_{\sigma} \lambda_{2}(y) B_{1, x} B_{2, y}(E(x-y)) \mathrm{d} \tau(y), \\
& g_{2}(x)=\oint_{\sigma} \lambda_{1}(y) B_{2, x} B_{1, y}(E(x-y)) \mathrm{d} \tau(y)+\oint_{\sigma} \lambda_{2}(y) B_{2, x} B_{2, y}(E(x-y)) \mathrm{d} \tau(y),
\end{aligned}
$$

where $\oint$ denotes the principal Cauchy value.

(5) For $\sigma=\{(s, 0) \times\{0\},-1<s<1\}, g_{1}(x)=0$, and $g_{2}(x)=V$ (with $V$ a constant), $\lambda_{1}$ and $\lambda_{2}$ are given by

$$
\begin{aligned}
& \lambda_{1}(s)=0, \forall(s, 0) \in \sigma, \\
& \lambda_{2}(s)=\frac{4}{(1-\nu)(3+\nu)} V \sqrt{1-s^{2}}, \forall(s, 0) \in \sigma .
\end{aligned}
$$

Proof. We denote by $\widetilde{\omega}^{\prime}$ the exterior domain $\widetilde{\omega}^{\prime}=\mathbb{R}^{2} \backslash \overline{\widetilde{\omega}}$.

We introduce the two following spaces

$$
H^{2}\left(\Delta^{2}, \widetilde{\omega}\right)=\left\{u \in H^{2}(\widetilde{\omega}), \Delta^{2} u \in L^{2}(\widetilde{\omega})\right\}, \quad W^{2}\left(\Delta^{2}, \widetilde{\omega}^{\prime}\right)=\left\{u \in W^{2}\left(\widetilde{\omega}^{\prime}\right), r^{2} \log (r) \Delta^{2} u \in L^{2}\left(\widetilde{\omega}^{\prime}\right)\right\},
$$

where $W^{2}\left(\widetilde{\omega}^{\prime}\right)$ is defined by (3.14) replacing $B$ by $\widetilde{\omega}$. We define the following bilinear forms:

$$
\begin{aligned}
& \widetilde{a}(u, v)=\int_{\widetilde{\omega}} \Delta u \Delta v+(1-\nu)\left(2 \frac{\partial^{2} u}{\partial x_{1} \partial x_{2}} \frac{\partial^{2} v}{\partial x_{1} \partial x_{2}}-\frac{\partial^{2} u}{\partial x_{1}^{2}} \frac{\partial^{2} v}{\partial x_{2}^{2}}-\frac{\partial^{2} u}{\partial x_{2}^{2}} \frac{\partial^{2} v}{\partial x_{1}^{2}}\right), \\
& \widetilde{a^{\prime}}(u, v)=\int_{\widetilde{\omega}^{\prime}} \Delta u \Delta v+(1-\nu)\left(2 \frac{\partial^{2} u}{\partial x_{1} \partial x_{2}} \frac{\partial^{2} v}{\partial x_{1} \partial x_{2}}-\frac{\partial^{2} u}{\partial x_{1}^{2}} \frac{\partial^{2} v}{\partial x_{2}^{2}}-\frac{\partial^{2} u}{\partial x_{2}^{2}} \frac{\partial^{2} v}{\partial x_{1}^{2}}\right) .
\end{aligned}
$$

Considering that $u \in H^{2}\left(\Delta^{2}, \widetilde{\omega}\right)$, the Green formula applied on $\widetilde{\omega}$ gives

$$
\widetilde{a}(u, v)=\int_{\widetilde{\omega}} \Delta^{2} u v-\int_{\partial \widetilde{\omega}}\left(B_{1}(u) v-B_{2}(u) \partial_{n} v\right), \forall v \in H^{2}(\widetilde{\omega}) .
$$

By the same reasoning on $u \in W^{2}\left(\Delta^{2}, \widetilde{\omega}^{\prime}\right)$ we have

$$
\widetilde{a^{\prime}}(u, v)=\int_{\widetilde{\omega}^{\prime}} \Delta^{2} u v+\int_{\partial \widetilde{\omega}}\left(B_{1}(u) v-B_{2}(u) \partial_{n} v\right) .
$$

Then we introduce the space

$$
K=\left\{u \in H^{2}\left(\Delta^{2}, \widetilde{\omega}\right) / \mathbb{P}_{1} \times W^{2}\left(\Delta^{2}, \widetilde{\omega}^{\prime}\right) / \mathbb{P}_{1}, \operatorname{supp}\left(\Delta^{2} u\right)=\sigma,\left[B_{1}(u)\right]_{\sigma}=\left[B_{2}(u)\right]_{\sigma}=0,[u]_{\tilde{\sigma} \backslash \bar{\sigma}}=\left[\partial_{n} u\right] \widetilde{\sigma} \backslash \bar{\sigma}=0\right\}
$$


and by using the regularity of $H_{l o c}^{2}(\Lambda)$ functions, $K$ rewrites as

$$
K=\left\{u \in W^{2}\left(\Delta^{2}, \Lambda\right) / \mathbb{P}_{1}, \operatorname{supp}\left(\Delta^{2} u\right)=\sigma,\left[B_{1}(u)\right]_{\sigma}=\left[B_{2}(u)\right]_{\sigma}=0\right\}
$$

$\left(\mathcal{R}_{\text {ext }}\right)$ reformulates as

$$
\text { find } R \in K \text { such as } B_{1}(R)=g_{1} \text { and } B_{2}(R)=g_{2} \text { on } \sigma \quad\left(\mathcal{R}_{\text {ext }}\right)
$$

and the variational formulation of $\left(\mathcal{R}_{\text {ext }}\right)$ is: find $R \in K$ such as

$$
a(R, v)=l(v), \forall v \in K,
$$

where

$$
\begin{aligned}
a(R, v) & =\int_{\Lambda} \Delta R \Delta v+(1-\nu)\left(2 \frac{\partial^{2} R}{\partial x_{1} \partial x_{2}} \frac{\partial^{2} v}{\partial x_{1} \partial x_{2}}-\frac{\partial^{2} R}{\partial x_{1}^{2}} \frac{\partial^{2} v}{\partial x_{2}^{2}}-\frac{\partial^{2} R}{\partial x_{2}^{2}} \frac{\partial^{2} v}{\partial x_{1}^{2}}\right), \\
l(v) & =\int_{\sigma} g_{1}[v]-g_{2}\left[\partial_{n} v\right] .
\end{aligned}
$$

Problem $\left(\mathcal{R}_{\text {ext }}\right)$ admits a unique solution in $K$. Indeed, it is coercive on $K$ : first we have

$$
a(u, u) \geq(1-\nu)|u|_{W^{2}(\Lambda)}^{2}, \forall u \in K
$$

and from Lemma A.1 we have:

$$
\|u\|_{K}=\|u\|_{W^{2}(\Lambda) / \mathbb{P}_{1}} \leq C(\Lambda)|u|_{W^{2}(\Lambda)}
$$

which gives the proof of the coercivity of $a(u, v)$ on $K$. After that the problem is continuous on $K$ : applying Lemma C.1, we obtain

$$
|l(v)| \leq C\left(\left\|g_{1}\right\|_{H_{00}^{3 / 2}(\sigma)^{\prime}}+\left\|g_{2}\right\|_{H_{00}^{1 / 2}(\sigma)^{\prime}}\right)\|v\|_{W^{2}(\Lambda) / \mathbb{P}_{1}}
$$

which implies the continuity of $l(v)$ on $K$. From (C.6) and (C.7) we deduce the continuity of the map $\left(g_{1}, g_{2}\right) \mapsto R$ from $H_{00}^{3 / 2}(\sigma)^{\prime} \times H_{00}^{3 / 2}(\sigma)^{\prime}$ into $W^{2}(\Lambda) / \mathbb{P}_{1}$.

Then as $K$ is a closed subspace of $W^{2}(\Lambda) / \mathbb{P}_{1}$ which is an Hilbert space, we deduce that it is an Hilbert space too. Thanks to Lax-Milgram's theorem, we get the well-possedness of problem $\left(\mathcal{R}_{\text {ext }}\right)$. Thus we define the following isomorphism

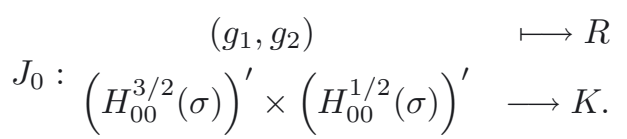

Let us consider the following problem: for $\left(q_{1}, q_{2}\right) \in H_{00}^{3 / 2}(\sigma) \times H_{00}^{1 / 2}(\sigma)$

$$
\text { find } Q \in K \text { such as }[Q]=q_{1} \text { and }\left[\partial_{n} Q\right]=q_{2} \text {. }
$$

Let $v \in K$, we have

$$
a(u, v)=\int_{\Lambda} \Delta^{2} v u+\int_{\sigma} B_{1}(v)[u]-B_{2}(v)\left[\partial_{n} u\right] .
$$

The variational formulation of $\left(\mathcal{Q}_{\text {ext }}\right)$ is: find $Q \in K$ such as

$$
a(Q, v)=l^{\prime}(v), \forall v \in K
$$

where

$$
l^{\prime}(v)=\int_{\sigma} q_{1} B_{1}(v)-\int_{\sigma} q_{2} B_{2}(v)
$$


In the same way as for problem $\left(\mathcal{R}_{\text {ext }}\right)$, we show the coercivity of problem $\left(\mathcal{Q}_{\text {ext }}\right)$. From Lemma C.2,

$$
l^{\prime}(v) \leq C\left(\left\|q_{1}\right\|_{H_{00}^{3 / 2}(\sigma)}+\left\|q_{2}\right\|_{H_{00}^{1 / 2}(\sigma)}\right)|v|_{H^{2}(B \backslash \bar{\sigma})} .
$$

From the equivalency of $W^{2}(\Lambda) / \mathbb{P}_{1}$-norm and seminorm, we deduce the continuity of the map $l^{\prime}(v)$ on $K$. Thanks to Lax-Milgram theorem we obtain the well-possedness of problem $\left(\mathcal{Q}_{\text {ext }}\right)$. Thus we can define the isomorphism

$$
\begin{array}{cc}
\left(q_{1}, q_{2}\right) & \longmapsto Q \\
J_{1}:\left(H_{00}^{3 / 2}(\sigma)\right) \times\left(H_{00}^{1 / 2}(\sigma)\right) & \longrightarrow K .
\end{array}
$$

We denote by $J=J_{1}^{-1} \circ J_{0}$ the isomorphism

$$
\begin{array}{cl}
\left(g_{1}, g_{2}\right) & \longmapsto\left(q_{1}, q_{2}\right) \\
J:\left(H_{00}^{3 / 2}(\sigma)^{\prime}\right) \times\left(H_{00}^{1 / 2}(\sigma)^{\prime}\right) & \longrightarrow\left(H_{00}^{3 / 2}(\sigma)\right) \times\left(H_{00}^{1 / 2}(\sigma)\right) .
\end{array}
$$

Now, let $\overline{\bar{u}}$ defined by

$$
\begin{aligned}
\overline{\bar{u}}(x) & =\int_{\sigma} \lambda_{1}(y) B_{1, y} E(x-y) \mathrm{d} \tau(y)+\int_{\sigma} \lambda_{2}(y) B_{2, y} E(x-y) \mathrm{d} \tau(y) \\
& =\int_{\partial \widetilde{\omega}} \widetilde{\lambda_{1}}(y) B_{1, y} E(x-y) \mathrm{d} \tau(y)+\int_{\partial \widetilde{\omega}} \widetilde{\lambda_{2}}(y) B_{2, y} E(x-y) \mathrm{d} \tau(y),
\end{aligned}
$$

where $x \in \Lambda$ and $\lambda_{1} \in H_{00}^{3 / 2}(\sigma), \lambda_{2} \in H_{00}^{1 / 2}(\sigma)$ and $\widetilde{\lambda_{i}} \in H^{3 / 2-(i-1)}(\widetilde{\sigma})$ defined by

$$
\tilde{\lambda}_{i}=\left\{\begin{array}{l}
\lambda_{i}, \text { on } \sigma \\
0, \text { on } \widetilde{\sigma} \backslash \bar{\sigma}
\end{array}\right.
$$

for $i \in\{1,2\}$. Firstly, $\overline{\bar{u}}$ is biharmonic. Indeed for $x \in \Lambda$ and $y \in \sigma$ the maps $y \mapsto B_{1, y} E(x-y)$ and $y \mapsto$ $B_{2, y} E(x-y)$ are $C^{\infty}(\sigma)$. Besides

$$
\Delta_{x}^{2} B_{1, y} E(x-y)=B_{1, y} \Delta_{x}^{2} E(x-y)=0 \quad \text { and } \quad \Delta_{x}^{2} B_{2, y} E(x-y)=B_{2, y} \Delta_{x}^{2} E(x-y)=0 .
$$

Then by using the regularity of $y \mapsto B_{1, y} E(x-y)$ and of $y \mapsto B_{2, y} E(x-y)$ we can interchange the integral and bilaplacian operators which leads to the following equation:

$$
\Delta^{2} \overline{\bar{u}}(x)=0, \forall x \in \Lambda
$$

Besides for $|x| \rightarrow \infty$, a Taylor expansion at x gives $\overline{\bar{u}}(x)=O(\log (|x|))$. A twice differentiation of $\overline{\bar{u}}$ and a Taylor expansion at point $x$ of $\overline{\bar{u}}$, show that $\overline{\bar{u}} \in W^{2}(\Lambda)$. Considering $\overline{\bar{u}}$ as an element of $W^{2}(\Lambda) / \mathbb{P}_{1}$, we get $\overline{\bar{u}} \in K$. By using jump relations given in ([9], pp. 385-386) we have

$$
\begin{aligned}
& B_{1, x}(\overline{\bar{u}})(x)=\oint_{\sigma} \lambda_{1}(y) B_{1, x} B_{1, y} E(x-y) \mathrm{d} \tau(y)+\oint_{\sigma} \lambda_{2}(y) B_{1, x} B_{2, y} E(x-y) \mathrm{d} \tau(y), \\
& B_{2, x}(\overline{\bar{u}})(x)=\oint_{\sigma} \lambda_{2}(y) B_{2, x} B_{1, y} E(x-y) \mathrm{d} \tau(y)+\oint_{\sigma} \lambda_{2}(y) B_{2, x} B_{2, y} E(x-y) \mathrm{d} \tau(y) .
\end{aligned}
$$

We show now that these two boundary integral equations describe the isomorphism $J^{-1}$. Indeed by using ([9], p. 384), we get

$$
\begin{aligned}
& \overline{\bar{u}}^{ \pm}(x)= \pm \lambda_{1}(x)+\oint_{\sigma} \lambda_{1}(y) B_{1, y} E(x-y) \mathrm{d} \tau(y)+\oint_{\sigma} \lambda_{2}(y) B_{2, y} E(x-y) \mathrm{d} \tau(y), \\
& \overline{\bar{u}}^{ \pm}(x)=\mp \lambda_{2}(x)+\oint_{\sigma} \lambda_{1}(y) \partial_{n_{x}} B_{1, y} E(x-y) \mathrm{d} \tau(y)+\oint_{\sigma} \lambda_{2}(y) \partial_{n_{x}} B_{2, y} E(x-y) \mathrm{d} \tau(y) .
\end{aligned}
$$


Then by difference

$$
[\overline{\bar{u}}]=\lambda_{1}, \quad\left[\partial_{n} \overline{\bar{u}}\right] \quad=-\lambda_{2} .
$$

As $J_{1}$ is an isomorphism, if we set $\lambda_{1}=[R]$ and $\lambda_{2}=\left[\partial_{n} R\right]$, we get $\overline{\bar{u}}=R$. Thus (C.8) with $B_{1}(\overline{\bar{u}})=g_{1}$ and $B_{2}(\overline{\bar{u}})=g_{2}$ defines the isomorphism $J^{-1}$. This ends the proof of the second, third and fourth points of the theorem. Now we must solve the two boundary integral equations (C.4) in order to prove the fifth point. We first compute the kernels in the simple case of a straight crack: we set $n=(0,1), x=(s, 0)$, and $y=(t, 0) \in \sigma$ and so:

$$
\begin{aligned}
& B_{1, x}\left(B_{1, y}(E(x-y))\right)=-\frac{3(-1+\nu)(3+\nu)}{2 \pi(s-t)^{4}}, \\
& B_{2, x}\left(B_{1, y}(E(x-y))\right)=B_{1, x}\left(B_{2, y}(E(x-y))\right)=0, \\
& B_{2, x}\left(B_{2, y}(E(x-y))\right)=\frac{(-1+\nu)(3+\nu)}{4 \pi(s-t)^{2}} .
\end{aligned}
$$

Integral equations (C.4a) and (C.4b) rewrite as

$$
\begin{aligned}
0 & =g_{1}(x)=-\oint_{-1}^{1} \lambda_{1}(t) \frac{3(-1+\nu)(3+\nu)}{2 \pi(s-t)^{4}} \mathrm{~d} t, \\
V & =g_{2}(x)=\oint_{-1}^{1} \lambda_{2}(t) \frac{(-1+\nu)(3+\nu)}{4 \pi(s-t)^{2}} \mathrm{~d} t .
\end{aligned}
$$

These two equations are uncoupled. For the first one the choice $\lambda_{1}=0$ is a solution, and by uniqueness it is the solution of (C.4a). For the second equation, we define the function:

$$
g(s)=\frac{1}{\pi} \oint_{-1}^{1} \frac{\lambda_{2}(t)}{s-t},-1<s<1 .
$$

We remark that (C.4b) rewrites as

$$
g^{\prime}(s)=-\frac{4}{(-1+\nu)(3+\nu)} V=A .
$$

Then we get $g(s)=A s+B$ with $B$ an arbitrary constant that we can take equal to 0 . From [12], $\lambda_{2}$ is given by the following formula

$$
\begin{aligned}
\lambda_{2}(s) & =-\frac{1}{\pi} \oint_{-1}^{1} \sqrt{\frac{1-t^{2}}{1-s^{2}}} \frac{A t}{s-t} \mathrm{dt}+\frac{C}{\sqrt{1-s^{2}}}=-\frac{1}{\pi \sqrt{1-s^{2}}} \oint_{1}^{1} \frac{\sqrt{1-t^{2}}}{s-t}(A(t-s)+A s) \mathrm{d} t+\frac{C}{\sqrt{1-s^{2}}} \\
& =\frac{-A}{\pi \sqrt{1-s^{2}}}\left(s \oint_{-1}^{1} \frac{\sqrt{1-t^{2}}}{s-t} \mathrm{~d} t-\oint_{-1}^{1} \sqrt{1-t^{2}} \mathrm{~d} t\right)+\frac{C}{\sqrt{1-s^{2}}} \\
& =\frac{-A}{\pi \sqrt{1-s^{2}}}\left(s^{2} \pi-\frac{\pi}{2}\right)+\frac{C}{\sqrt{1-s^{2}}}=A \sqrt{1-s^{2}},
\end{aligned}
$$

where we set $C=A / 2$ in order to verify $\lambda_{2}(-1)=\lambda_{2}(1)=0$ (ie $\lambda_{2} \in H_{00}^{1 / 2}(\sigma)$ ). This ends the proof of the theorem.

Lemma C.4. Let $R$ be the solution of the exterior problem $(\mathcal{R})$ given in (C.1), we have the following asymptotic behavior:

For $|x| \rightarrow \infty$

$$
|R(x)| \leq C \log (|x|), \quad|\nabla R(x)| \leq \frac{C}{|x|}, \quad\left|\nabla^{2} R(x)\right| \leq \frac{C}{|x|^{2}}
$$


For $\epsilon \rightarrow 0$

$$
\left\|R\left(\frac{x}{\epsilon}\right)\right\|_{0, \Omega_{\epsilon}}=O(-\log (\epsilon)), \quad\left|R\left(\frac{x}{\epsilon}\right)\right|_{1, \Omega_{\epsilon}}=O(\sqrt{-\log (\epsilon)}), \quad \mid R\left(\frac{x}{\epsilon}\right) \|_{2, \Omega_{\epsilon}}=O\left(\frac{1}{\epsilon}\right)
$$

Proof. From the expression of $R$ given in Theorem C.3 and by using a Taylor expansion at point $x$ and taking $|x| \rightarrow+\infty$, we get the first inequality. For the second and the third ones, we must differentiate the expression of $R \in C^{\infty}\left(\mathbb{R}^{2} \backslash \bar{\sigma}\right)$, interchange integral and derivative, and use a Taylor expansion around $x$. We refer to Lemma B.5 for the proof of the three last estimates.

Lemma C.5. Let be $w_{\epsilon}$ the solution of problem $\left(\mathcal{Q}_{\epsilon}^{c}\right)$ given in (4.6), and $R$ the solution of the exterior problem $\left(\mathcal{R}_{\text {ext }}\right)$ given in (4.7), then we have the following asymptotic expansion:

$$
w_{\epsilon}=\epsilon^{2} R\left(\frac{x}{\epsilon}\right)+e_{\epsilon}
$$

with

$$
\begin{aligned}
\left\|e_{\epsilon}\right\|_{2, \Omega_{\epsilon}} & =O\left(-\epsilon^{2} \log (\epsilon)\right), \\
\left|w_{\epsilon}\right|_{2, \Omega_{\epsilon}} & =O(\epsilon) .
\end{aligned}
$$

Proof. $e_{\epsilon}$ solves the following problem

$$
\left(\mathcal{E}_{\epsilon}\right)\left\{\begin{aligned}
\Delta^{2} e_{\epsilon}-\gamma \Delta e_{\epsilon}+e_{\epsilon} & =\gamma \Delta R\left(\frac{x}{\epsilon}\right)-\epsilon^{2} R\left(\frac{x}{\epsilon}\right) & & \text { on } \Omega_{\epsilon} \\
B_{1}\left(e_{\epsilon}\right)-\gamma \partial_{n} e_{\epsilon} & =-B_{1}\left(v_{0}\right)-\underbrace{\frac{1}{\epsilon} B_{1}(R)\left(\frac{x}{\epsilon}\right)}_{=0}-\gamma \partial_{n} v_{0}=\varphi_{1}(x)=O(1), & & \text { on } \sigma_{\epsilon} \\
B_{2}\left(e_{\epsilon}\right) & =-B_{2}\left(v_{0}\right)-g_{2}\left(\frac{x}{\epsilon}\right)=\varphi_{2}(x)=O(x), & & \text { on } \sigma_{\epsilon} \\
B_{1}\left(e_{\epsilon}\right)-\gamma \partial_{n} e_{\epsilon} & =-\frac{1}{\epsilon} B_{1}(R)\left(\frac{x}{\epsilon}\right)=\phi_{1}(x)=O\left(\frac{\epsilon^{2}}{|x|^{3}}\right), & & \text { on } \partial \Omega \\
B_{2}\left(e_{\epsilon}\right) & =-B_{2}(R)\left(\frac{x}{\epsilon}\right)=\phi_{2}(x)=O\left(\frac{\epsilon^{2}}{|x|^{2}}\right), & & \text { on } \partial \Omega
\end{aligned}\right.
$$

where $g_{2}(X)=-\frac{\partial^{2} v_{0}}{\partial x_{2}^{2}}(0)-\nu \frac{\partial^{2} v_{0}}{\partial x_{1}^{2}}(0)=-\beta$ for $X \in \sigma$.

The variational formulation of problem $\left(\mathcal{E}_{\epsilon}\right)$ given in (C.9) is: find $e_{\epsilon} \in H^{2}\left(\Omega_{\epsilon}\right)$ such as

$$
a_{\epsilon}\left(e_{\epsilon}, v\right)=\int_{\Omega_{\epsilon}} \gamma \Delta R\left(\frac{x}{\epsilon}\right) v-\epsilon^{2} R\left(\frac{x}{\epsilon}\right) v+\int_{\sigma_{\epsilon}} \varphi_{1}[v]-\varphi_{2}\left[\partial_{n} v\right]-\int_{\Gamma}\left(\phi_{1} v-\phi_{2} \partial_{n} v\right), \forall v \in H^{2}\left(\Omega_{\epsilon}\right) .
$$

Integrating by parts the first term of $a_{\epsilon}\left(e_{\epsilon}, v\right)$ gives

$$
\int_{\Omega_{\epsilon}} \Delta R\left(\frac{x}{\epsilon}\right) v=\epsilon \int_{\Gamma} \partial_{n} R\left(\frac{x}{\epsilon}\right) v-\epsilon \int_{\sigma_{\epsilon}}\left[\partial_{n} R\left(\frac{x}{\epsilon}\right) v\right]-\epsilon^{2} \int_{\Gamma} R\left(\frac{x}{\epsilon}\right) \partial_{n} v+\epsilon^{2} \int_{\sigma_{\epsilon}}\left[R\left(\frac{x}{\epsilon}\right) \partial_{n} v\right]+\int_{\Omega_{\epsilon}} \epsilon^{2} R\left(\frac{x}{\epsilon}\right) \Delta v .
$$

The variational formulation of $\left(\mathcal{E}_{\epsilon}\right)$ rewrites as:

$$
\begin{aligned}
a_{\epsilon}\left(e_{\epsilon}, v\right)= & \epsilon \int_{\Gamma} \partial_{n} R\left(\frac{x}{\epsilon}\right) v-\epsilon \int_{\sigma_{\epsilon}}\left[\partial_{n} R\left(\frac{x}{\epsilon}\right) v\right]-\epsilon^{2} \int_{\Gamma} R\left(\frac{x}{\epsilon}\right) \partial_{n} v \\
& +\epsilon^{2} \int_{\sigma_{\epsilon}}\left[R\left(\frac{x}{\epsilon}\right) \partial_{n} v\right]+\int_{\Omega_{\epsilon}} \epsilon^{2} R\left(\frac{x}{\epsilon}\right) \Delta v-\epsilon^{2} R\left(\frac{x}{\epsilon}\right) v \\
& +\int_{\sigma_{\epsilon}} \varphi_{1}[v]-\varphi_{2}\left[\partial_{n} v\right]-\int_{\Gamma}\left(\phi_{1} v-\phi_{2} \partial_{n} v\right), \forall v \in H^{2}\left(\Omega_{\epsilon}\right) .
\end{aligned}
$$

We split $e_{\epsilon}$ in the distributionnal sense into the sum $e_{\epsilon}=e_{\epsilon}^{1,+}+e_{\epsilon}^{1,-}+e_{\epsilon}^{2}$ with 
- $e_{\epsilon}^{1, \pm} \in H^{2}\left(\Omega_{\epsilon}\right) / \mathbb{P}_{1}$ defined by

$$
b_{\epsilon}\left(e_{\epsilon}^{1, \pm}, v\right)=l_{\epsilon}^{1, \pm}(v), \forall v \in H^{2}\left(\Omega_{\epsilon}\right) / \mathbb{P}_{1}, \text { where } b_{\epsilon} \text { is given in }(\mathrm{B} .16)
$$

and

$$
l_{\epsilon}^{1, \pm}(v)=-\gamma \epsilon \int_{\sigma_{\epsilon}}\left(\partial_{n} R\left(\frac{x}{\epsilon}\right) v\right)^{ \pm}+\gamma \epsilon^{2} \int_{\sigma_{\epsilon}}\left(R\left(\frac{x}{\epsilon}\right) \partial_{n} v\right)^{ \pm}
$$

where we recall that for $x \in \sigma_{\epsilon}, u(x)^{ \pm}$are the right and left limit values (see Fig. 2).

We check easily that each problem defining the solution $e_{\epsilon}^{1, \pm}$ is well defined on $H^{2}\left(\Omega_{\epsilon}\right) / \mathbb{P}_{1}$.

- $e_{\epsilon}^{2} \in H^{2}\left(\Omega_{\epsilon}\right)$ is defined by the variational form:

$$
\begin{aligned}
a_{\epsilon}\left(e_{\epsilon}^{2}, v\right)= & a_{\epsilon}\left(e_{\epsilon}-e_{\epsilon}^{1,+}-e_{\epsilon}^{1,-}, v\right)=-\int_{\Omega_{\epsilon}} \epsilon^{2} R\left(\frac{x}{\epsilon}\right) v+\int_{\Omega_{\epsilon}} \gamma \epsilon^{2} R\left(\frac{x}{\epsilon}\right) \Delta v-\gamma \int_{\Omega_{\epsilon}} \nabla\left(e_{\epsilon}^{1,+}+e_{\epsilon}^{1,-}\right) . \nabla v \\
& -\int_{\Omega_{\epsilon}}\left(e_{\epsilon}^{1,+}+e_{\epsilon}^{1,-}\right) v-\int_{\Gamma}\left(\phi_{1} v-\phi_{2} \partial_{n} v\right)+\int_{\sigma_{\epsilon}} \varphi_{1}[v]-\varphi_{2}\left[\partial_{n} v\right], \forall v \in H^{2}\left(\Omega_{\epsilon}\right) .
\end{aligned}
$$

As for $e_{\epsilon}^{1, \pm}$, we can show that $e_{\epsilon}^{2}$ is well defined.

Now let us estimate $\left\|e_{\epsilon}^{1, \pm}\right\|_{H^{2}\left(\Omega_{\epsilon}\right) / \mathbb{P}_{1}}$ and $\left\|e_{\epsilon}^{2}\right\|_{2, \Omega_{\epsilon}}$. A change of variable, the trace theorem on $B \backslash \overline{\widetilde{\omega}}$, the equivalency of $H^{2}(B \backslash \overline{\widetilde{\omega}}) / \mathbb{P}_{1}$-norm and seminorm and a change of variable again lead to

$$
\begin{aligned}
C\left|e_{\epsilon}^{1,+}\right|_{2, \Omega_{\epsilon}}^{2} \leq b_{\epsilon}\left(e_{\epsilon}^{1,+}, e_{\epsilon}^{1,+}\right) & =-\epsilon \int_{\sigma_{\epsilon}}\left(\partial_{n} R\left(\frac{x}{\epsilon}\right) e_{\epsilon}^{1,+}\right)^{+}+\epsilon^{2} \int_{\sigma_{\epsilon}}\left(R\left(\frac{x}{\epsilon}\right) \partial_{n} e_{\epsilon}^{1,+}\right)^{+} \\
& =-\epsilon^{2} \int_{\sigma}\left(\partial_{n} R(X) e_{\epsilon}^{1,+}(\epsilon X)\right)^{+}+\epsilon^{3} \int_{\sigma}\left(R(X) \partial_{n} e_{\epsilon}^{1,+}((\epsilon X))^{+}\right. \\
& \leq C \epsilon^{2}\left\|e_{\epsilon}^{1,+}(\epsilon X)\right\|_{H_{00}^{3 / 2}(\sigma) / \mathbb{P}_{1}}+C \epsilon^{3}\left\|\partial_{n} e_{\epsilon}^{1,+}(\epsilon X)\right\|_{H_{00}^{1 / 2}(\sigma) / \mathbb{P}_{1}} \\
& \leq C \epsilon^{2} \mid e_{\epsilon}^{1,+}(\epsilon X)\left\|_{H^{3 / 2}(\partial \widetilde{\omega}) / \mathbb{P}_{1}}+C \epsilon^{3}\right\| \partial_{n} e_{\epsilon}^{1,+}(\epsilon X) \|_{H^{1 / 2}(\partial \widetilde{\omega}) / \mathbb{P}_{1}} \\
& \leq \epsilon^{2}\left\|e_{\epsilon}^{1,+}(\epsilon X)\right\|_{H^{2}(B \backslash \bar{\omega}) / \mathbb{P}_{1}} \leq C \epsilon^{2}\left|e_{\epsilon}^{1,+}(\epsilon X)\right|_{2, B \backslash \widetilde{\omega}} \leq C \epsilon^{3}\left|e_{\epsilon}^{1,+}\right|_{2, \Omega_{\epsilon}} .
\end{aligned}
$$

We deduce that $\left|e_{\epsilon}^{1,+}\right|_{2, \Omega_{\epsilon}}=O\left(\epsilon^{3}\right)$. In the same way, applying the trace theorem on $\widetilde{\omega}$ we get $\left|e_{\epsilon}^{1,-}\right|_{2, \Omega_{\epsilon}}=$ $O\left(\epsilon^{3}\right)$.

As $e_{\epsilon}^{1, \pm}$ is defined up to a polynomial of degree less or equal than 1, and from Lemma C.1 and the estimates $\left\|\phi_{1}\right\|_{-3 / 2, \Gamma}=O\left(\epsilon^{2}\right),\left\|\phi_{2}\right\|_{-1 / 2, \Gamma}=O\left(\epsilon^{2}\right),\left|\varphi_{1}(\epsilon X)\right|=O(1),\left|\varphi_{2}(\epsilon X)\right|=O(\epsilon)$ and $\left|e_{\epsilon}^{1, \pm}\right|_{2, \Omega_{\epsilon}}=O\left(\epsilon^{3}\right)$ and a change of variable we have

$$
\begin{aligned}
C\left\|e_{\epsilon}^{2}\right\|_{2, \Omega_{\epsilon}}^{2} \leq a_{\epsilon}\left(e_{\epsilon}^{2}, e_{\epsilon}^{2}\right) \leq & \epsilon^{2}\left\|R\left(\frac{x}{\epsilon}\right)\right\|_{0, \Omega_{\epsilon}}\left\|e_{\epsilon}^{2}\right\|_{0, \Omega_{\epsilon}}+\gamma \epsilon^{2}\left\|R\left(\frac{x}{\epsilon}\right)\right\|_{0, \Omega_{\epsilon}}\left|e_{\epsilon}^{2}\right|_{2, \Omega_{\epsilon}} \\
& +\gamma\left(\left|e_{\epsilon}^{1,+}\right|_{H^{1}\left(\Omega_{\epsilon}\right) / \mathbb{P}_{1}}+\left|e_{\epsilon}^{1,-}\right|_{H^{1}\left(\Omega_{\epsilon}\right) / \mathbb{P}_{1}}\right)\left|e_{\epsilon}^{2}\right|_{1, \Omega_{\epsilon}} \\
& +\left(\left\|e_{\epsilon}^{1,+}\right\|_{L^{2}\left(\Omega_{\epsilon}\right) / \mathbb{P}_{1}}+\left\|e_{\epsilon}^{1,-}\right\|_{L^{2}\left(\Omega_{\epsilon}\right) / \mathbb{P}_{1}}\right)\left\|e_{\epsilon}^{2}\right\|_{0, \Omega_{\epsilon}} \\
& +\left\|\phi_{1}\right\|_{-3 / 2, \Gamma}\left\|e_{\epsilon}^{2}\right\|_{3 / 2, \Gamma}+\left\|\phi_{2}\right\|_{-1 / 2, \Gamma}\left\|\partial_{n} e_{\epsilon}^{2}\right\|_{1 / 2, \Gamma} \\
& +\epsilon\left\|\varphi_{1}(\epsilon X)\right\|_{H_{00}^{3 / 2}(\sigma)^{\prime}}\left|e_{\epsilon}^{2}(\epsilon X)\right|_{2, B \backslash \bar{\sigma}}+\left\|\varphi_{2}(\epsilon X)\right\|_{H_{00}^{1 / 2}(\sigma)^{\prime}}\left|e_{\epsilon}^{2}(\epsilon X)\right|_{2, B \backslash \bar{\sigma}} \\
\leq & C\left(-\epsilon^{2} \log (\epsilon)+C \epsilon^{3}+C \epsilon^{2}+C \epsilon^{2}\right)\left\|e_{\epsilon}^{2}\right\|_{2, \Omega_{\epsilon}} .
\end{aligned}
$$

We deduce that

$$
\left\|e_{\epsilon}^{2}\right\|_{2, \Omega_{\epsilon}}=O\left(-\epsilon^{2} \log (\epsilon)\right)
$$

We come back to $e_{\epsilon}$ : as $e_{\epsilon}^{1, \pm}$ is defined up to a polynomial of degree less or equal than 1 and thanks to the Deny-Lions inequality given in (B.17) we have

$$
\left\|e_{\epsilon}\right\|_{2, \Omega_{\epsilon}} \leq\left\|e_{\epsilon}^{1,+}\right\|_{H^{2}\left(\Omega_{\epsilon}\right) / \mathbb{P}_{1}}+\left\|e_{\epsilon}^{1,-}\right\|_{H^{2}\left(\Omega_{\epsilon}\right) / \mathbb{P}_{1}}+\left\|e_{\epsilon}^{2}\right\|_{H^{2}\left(\Omega_{\epsilon}\right)}=O\left(-\epsilon^{2} \log (\epsilon)\right) .
$$


Finally, to estimate $\left|w_{\epsilon}\right|_{2, \Omega_{\epsilon}}$ we use Lemma C.4:

$$
\left|w_{\epsilon}\right|_{2, \Omega_{\epsilon}} \leq \epsilon^{2}\left|R\left(\frac{x}{\epsilon}\right)\right|_{2, \Omega_{\epsilon}}+\left|e_{\epsilon}\right|_{2, \Omega_{\epsilon}} \leq C \epsilon+C \epsilon^{2} \log (\epsilon) \leq C \epsilon
$$

which ends the proof.

\section{REFERENCES}

[1] R.A. Adams, Sobolev spaces. Pure Appl. Math. Academic Press, New York (1978).

[2] S. Amstutz, Sensitivity analysis with respect to a local perturbation of the material property. Asymp. Anal. 49 (2006) 87-108.

[3] S. Amstutz, The topological asymptotic for the Navier-Stokes equations. ESAIM: COCV 11 (2010) 401-425.

[4] S. Amstutz and A.A. Novotny, Topological asymptotic analysis of the Kirchhoff plate bending problem. ESAIM: COCV 17 (2011) 705-721.

[5] G. Aubert and A. Drogoul, Topological gradient for fourth-order PDE and application to the detection of fine structures in 2D images. C.R. Math. 352 (2014) 609-613.

[6] D. Auroux, From restoration by topological gradient to medical image segmentation via an asymptotic expansion. Math. Comput. Model. 49 (2009) 2191-2205.

[7] D. Auroux, M. Masmoudi and L. Jaafar Belaid, Image restoration and classification by topological asymptotic expansion, in Variational Formulations in Mechanics: Theory and Applications. Edited by E. Taroco, E.A. de Souza Neto and A.A. Novotny. CIMNE, Barcelona, Spain (2007) 23-42.

[8] L. Jaafar Belaid, M. Jaoua, M. Masmoudi, and L. Siala, Application of the topological gradient to image restoration and edge detection. Eng. Anal. Boundary Elements 32 (2008) 891-899.

[9] G. Chen and J. Zhou, Boundary Element Methods with Applications to Nonlinear Problems. Atlantis Stud. Math. Eng. Sci. (1992).

[10] A. Drogoul, Numerical analysis of the topological gradient method for fourth order models and applications to the detection of fine structures in imaging. SIAM J. Imaging Sci. 7 (2014) 2700-2731.

[11] A. Drogoul, Topological gradient method applied to the detection of edges and fine structures in imaging. Ph.D. thesis, University of Nice Sophia Antipolis (2014).

[12] P.A. Martin, Exact solution of a hypersingular integral equation. J. Integral Equations Appl. 4 (1992) $197-204$.

[13] M. Masmoudi, The topological asymptotic. In Computational Methods for Control Applications. Vol. 16 of GAKUTO Internat. Ser. Math. Appl. Tokyo, Japan (2001).

[14] J.-C. Nédélec, Acoustic and electromagnetic equations: integral representations for harmonic problems. Appl. Math. Sci. Springer, New York (2001).

[15] L. Nirenberg, On elliptic partial differential equations. Annali della Scuola Normale Superiore di Pisa - Classe di Scienze 13 (1959) 115-162.

[16] D. Ruiz, A note on the uniformity of the constant in the Poincaré inequality. Preprint arXiv:1208.6045 (2012).

[17] J. Sokolowski and A. Zochowski, On the topological derivative in shape optimization. SIAM J. Control Optim. 37 (1999) 1251-1272.

[18] C. Steger, An unbiased detector of curvilinear structures. IEEE Trans. Pattern Anal. Mach. Intell. 20 (1998) $113-125$. 